\title{
On spiders from the tundra zone of the Kola Peninsula, Russia (Arachnida: Aranei)
}

\author{
О пауках тундровой зоны Кольского полуострова \\ (Arachnida: Aranei)
}

\author{
Andrei V. Tanasevitch ${ }^{1} \&$ Leonid B. Rybalov ${ }^{2}$

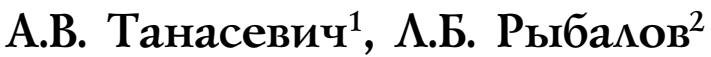

\begin{abstract}
${ }^{1}$ Centre for Forest Ecology and Production, Russian Academy of Sciences, Profsoyuznaya Str. 84/32, Moscow 117997, Russia. E-mail: and-tan@mail.ru.

Центр по проблемам экологии и продуктивности лесов РАН, Профсоюзная ул. 84/32, Москва 117997, Россия.

${ }^{2}$ Institute of Ecology and Evolution, Russian Academy of Sciences, Leninsky Prospect 33, Moscow 119071, Russia. E-mail: lrybalov52@mail.ru.

Институт проблем экологии и эволюции РАН, Ленинский проспект 33, Москва 119071, Россия.
\end{abstract}

KEY WORDS: Spiders, Russia, Kola Peninsula, tundra, chorology, new synonym.

КЛЮЧЕВЫЕ СЛОВА: Пауки, Россия, Кольский п-ов, тундра, хорология, новый синоним.

ABSTRACT. A checklist of 105 spiders from the tundra zone of the Kola Peninsula is based and compiled on the original collections and on available literature data. Oreoneta sinuosa (Tullgren, 1955) is reported as a new to the Russian fauna. A new synonym is proposed: Lepthyphantes murmanicola Strand, 1913, syn.n. = Oryphantes angulatus $(\mathrm{O}$. Pickard-Cambridge, 1881). Some previous misidentifications are rectified. Faunistic data and the distribution pattern are indicated for each species. For the majority of the recorded spiders, the northern coast of the Kola Peninsula is the northernmost point of their known distributions. The spider fauna of the tundra zone of the Kola Peninsula has a typical boreal face, and is composed of widespread or European boreal/polyzonal species. There are no real Arctic elements found here. The zonal plant communities have the fewest spider species, lack of the specific elements, and are comprised of boreal or ubiquitous species. Intrazonal and anthropogenic communities are much richer, and together with azonal communities serve as a shelter not only for the southern elements, but also for the arcto-alpine species.

РЕЗЮМЕ. Приведен аннотированный список 105 видов пауков тундровой зоны Кольского п-ова, составленный на основании недавних сборов и литературных данных. Вид Oreoneta sinuosa (Tullgren, 1955) впервые отмечен для фауны России. Lepthyphantes murmanicola Strand, 1913, syn.n. признан младшим синонимом Oryphantes angulatus (O. Pickard-Cambridge, 1881). Исправлены некоторые ошибочные определения предыдущих авторов. Для каждого вида приведены места находок и тип ареала. Для большинства отмеченных видов северное побережье Кольского п-ова является самой северной точкой распространения. Фауна тундр Кольского п-ова характеризуется отсутствием собственно арктических элементов, имеет типичный бореальный облик и составлена широкоареальными бореальными и полизональными видами. Зональные тундровые сообщества наиболее бедны в таксономическом плане и характеризуются отсутствием специфичных видов. Фауна интразональных, азональных и антропогенно модифицированных сообществ значительно богаче; именно эти типы сообществ служат основным прибежищем не только для южных фаунистических элементов, но и для немногочисленных арктоальпийских видов.

\section{Introduction}

The tundra landscape zone of the Kola Peninsula is a narrow belt ca $30-60 \mathrm{~km}$ wide (see Map). Owing to a strong vertical partitioning of the territory, numerous lakes and rivers, the tundra landscape is strongly fragmented. The zonal plant communities are represented by different types of the undershrub-mosslichen tundra vegetation which alternates with open water surfaces, sedge-moss bogs, peatbogs, Betula and Salix stands in depressions. The tops of hills and abrupt stony slopes are occupied by crustose lichen communities. The seashore is usual narrow, with salt marshes and meadow-like vegetation. Data on 55 spiders known from the tundra zone of Kola Peninsula are contained in two papers (Fedotov [1912] and Shokhin et al. [2004]). New material from Teriberka and Dal'niye Zelentsy has doubled the number of species in this list. 


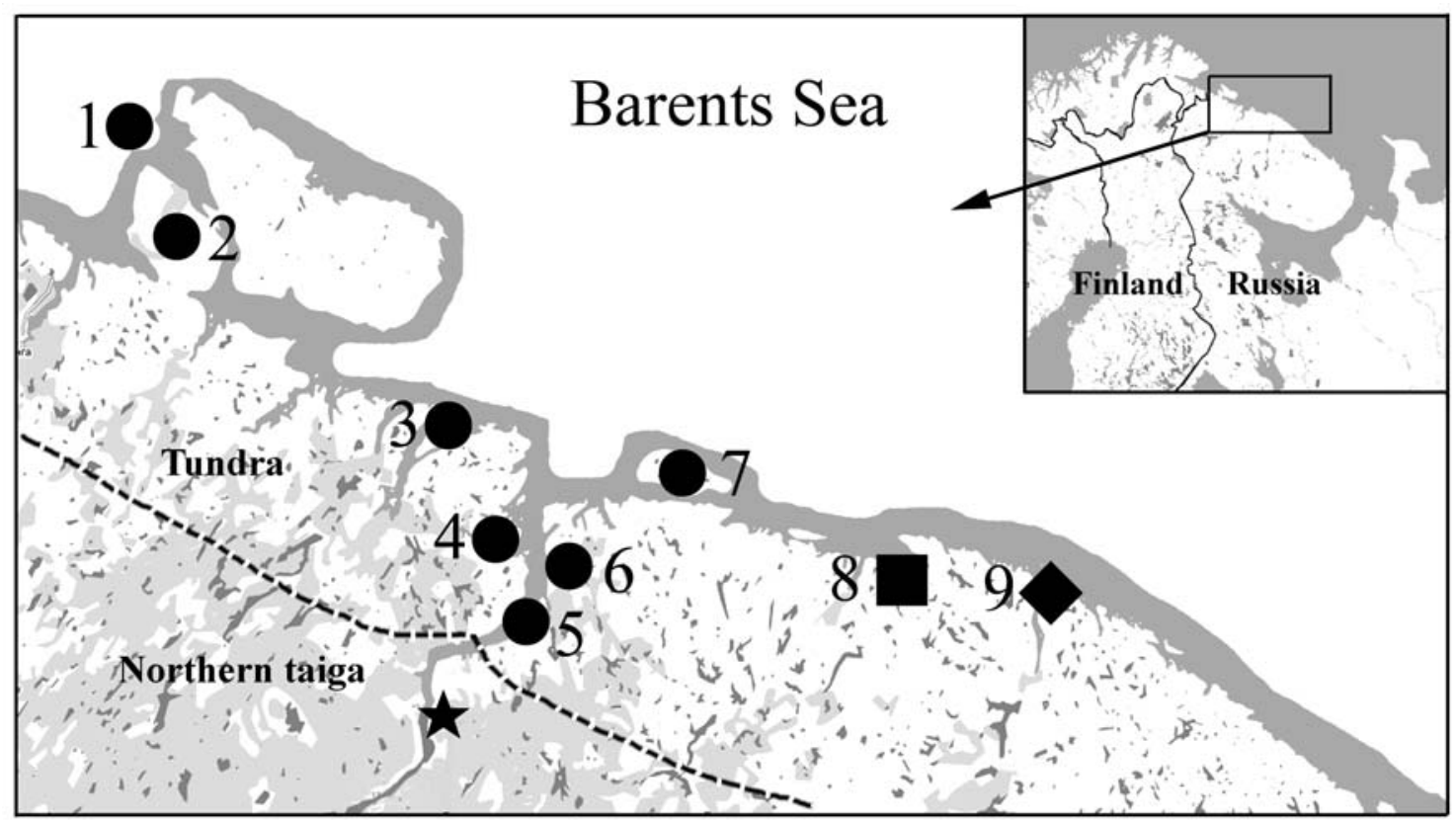

Map. Localities in Kola Peninsula: 1 - Bolshoy Aynovskiy Island; 2 - Rybachiy Peninsula; 3 - Shalim Island; 4 - Sedlovaty Island, Yekaterininskiy Island, Pala Bay, Olen'ya Bay, Murmansk Field Station; 5 - Srednyaya Bay; 6 — Tyuva Bay; 7 - Kildin Island; 8 - Teriberka, Lodeynoye, Logeynaya Bay, Korabelnaya Bay; 9 - Dal'niye Zelentsy; circle — literature data [Fedotov, 1912], square collecting by L. Rybalov, V. Semenov \& V. Piryugin; rhomb - collecting by A. Babenko; asterisk - Murmansk City; dotted line southern border of tundra landscape zone.

Карта. Точки сборов на Кольском полуострове: 1 - Большой Айновский о-в; 2 - Рыбачий п-ов; 3 - Шалим о-в; $4-$ Седловатый о-в, Екатерининский о-в, Пала губа, Оленья губа, Мурманская биологическая станция; 5 - Средняя губа; 6 - Тюва губа; 7 - Кильдин о-в; 8 - Териберка, Лодейное, Лодейная губа, Корабельная губа; 9 - Дальние Зеленцы; круг - литературные данные [Федотов, 1912], квадрат - сборы Л. Рыбалова, В. Семенова и В. Пирюгина; ромб - сборы А. Бабенко; звездочка - г. Мурманск; пунктирная линия - южная граница тундровой зоны.

\section{Material and methods}

This study is based mainly on the collections made recently in Teriberka (ca $69^{\circ} 9^{\prime} \mathrm{N}, 35^{\circ} 8^{\prime} \mathrm{E}$ ) by Leonid B. Rybalov and Victor B. Semenov in 3-11.VII.2008, as well as by Victor S. Piryugin in 3-7.VIII.2009. Some material was collected at Dal'niye Zelentsy (ca $69^{\circ} 7^{\prime} \mathrm{N}, 36^{\circ} 3^{\prime} \mathrm{E}$ ) by Anatoly B. Babenko in 4-26.VII. 2009. Spiders were collected by the sifting of moss and litter, soil sampling, pitfall trapping, and hand collecting. In addition, all available literature data on spiders of the northern coast of the Kola Peninsula is compiled. Linyphiids spiders recorded by Shokhin, et al. [2004] from Teriberka were examined. All material is stored in the personal collection of Andrei V. Tanasevitch, Moscow, Russia.

For study of the zonal-landscape distribution of tundra spiders we used the scheme proposed by Chernov [1978] for dividing plant communities in the Subarctic. Based on this classification, there are three main zonal types of communities in tundra (for more details, see Tanasevitch \& Koponen [2007]):

Zonal communities - plant communities distributed into one zone and which are situated at the watershed. Zonal communities define the vegetation zonal face of the landscape. For the Kola tundra, this is the polyvariant lichen-moss-undershrub-dwarf birch (or willow-dwarf birch) tundra. In zonal communities, the abiotic factors are more extreme than they are in the intrazonal communities, and living conditions therefore are more adverse.

Intrazonal communities - communities distributed in one or several zones, the typical representatives of this type are swamps and the river valley vegetation, e.g., willow stands on floodplains, meadows, pebble banks, rocky and/or grassy slopes, forest stands on river banks or river terraces, etc. Commonly, parts of intrazonal type of communities are situated on watersheds among zonal type of vegetation: there are willow stands in depressions, shrubby banks of lakes, flat-hill peatbogs with sedge fens or sphagnum bogs in hollows, grassy slopes of small brooks, etc. The intrazonal communities are smoothing gradient of the climatic factors (especially, temperature and humidity), making living conditions substantially better (less pessimal). Some rivers penetrate several zones/subzones and river valleys are powerful channels for the penetration of southern forms to the North and vice versa. Some of southern species can, using intrazonal biotopes, come to watersheds and become part of the tundra fauna.

Azonal communities - plant communities which not confined to a certain zone, but which are distributed in many zones; in the Kola tundra this type is seacoast meadows and marshes.

Anthropogenic communities - these kinds of communities are very common in the North today. It is not 
only anthropogenically changed natural vegetation (meadow formation sites, roadsides of roads, etc.), but also dump sites, dustbins, destroyed constructions, and so on.

Distributional pattern of species follows that suggested by Tanasevitch \& Koponen [2007] with a few minor changes.

Abbreviation used in text: Zonal, Intra, Azonal \& Anthro - zonal, intrazonal, azonal \& anthropogenic communities, respectively, L.R. - L. Rybalov, V.S. V. Semenov, V.P. - V. Piryugin.

\section{List of spiders}

\section{Fam. THERIDIIDAE (2)}

Robertus lividus (Blackwall, 1836)

2004 Robertus lividus - Shokhin et al.: 142.

MATERIAL. Intra: $3 \sigma^{7} \sigma^{7}$, Teriberka, Orlovka River valley, elfin Betula sp. sparse forest on gentle slope with Vaccinium myr tillus, Cornus suecica, Geranium sanguineum, Epilobium angustifolium, Calamagrostis sp., 9.VII.2008, leg. L.R. \& V. S; 1 ○', Orlovka River valley, elfin Betula sp. sparse forest on gentle slope with Vaccinium myrtillus, Gymnocarpium dryopteris, Cornus sueci$c a$, no moss or lichen, 9.VII.2008, leg. L.R. \& V.S. Azonal: $2 \sigma^{7} \sigma^{7}$ Dal'niye Zelentsy, meadow-like community on slope to sea, pitfall traps, 4-26.VII.2009, leg. A.B. Anthro: 1 , ruderal plant community with Poa sp., Deschampsia caespitosa, Epilobium angustifolium, etc., 3.VII.2008, leg. L.R. \& V.S.

PREVIOUS RECORDS. Teriberka [Shokhin et al., 2004].

COMMENTS. Teriberka is the northernmost known distribution of the species.

RANGE. Palaearctic-Alaskan boreo-nemoral.

\section{Robertus scoticus Jackson, 1914}

MATERIAL. Zonal: 3 + , Teriberka, near Tretie Titovskoye Lake, dwarf birch-lichen mountain tundra with Betula nana, Cladonia sp., 4-6.VIII.2009, leg. V.P. Intra: 1 +, elfin Betula sp. sparse forest on hill slope with Vaccinium myrtillus, Gymnocarpium dryopteris, Cornus suecica, Calamagrostis sp., no moss or lichen, 10.VII.2008, leg. L.R. \& V.S.; 1 , Betula tortuosa undergrowth on airfield with Empetrum sp., Vaccinium myrtillus, Pleurozium schreberi, Hylocomium sp., etc., 5.VII.2008, leg. L.R. \& V.S.; 1 o, same, 4-6.VIII.2009, leg. V.P. Anthro: 2 of, ruderal plant community with Poa sp., Deschampsia caespitosa, Epilobium angustifolium, etc., 3.VII.2008, leg. L.R. \& V.S.

COMMENTS. Teriberka is the northernmost known distribution of the species.

RANGE. Palaearctic boreo-nemoral.

Fam. LINYPHIIDAE (65)

Agyneta conigera (O. Pickard-Cambridge, 1863)

MATERIAL. Intra: 2 ㅇ, Teriberka, Orlovka River valley, elfin Betula sp. sparse forest on gentle slope with Vaccinium myrtillus, Cornus suecica, Geranium sanguineum, Epilobium angustifolium, Calamagrostis sp., 9.VII.2008, leg. L.R. \& V.S.

COMMENTS. Teriberka is the northernmost known distribution of the species.

RANGE. Palaearctic boreo-nemoral.
Agyneta mossica (Schikora, 1993)

MATERIAL. Intra: $1 \sigma^{r}$, Teriberka, Orlovka River valley, swampy meadow with Betula nana, Carex sp., Eriophorum sp., Vaccinium oxycoccus, Rubus chamaemorus, Menyanthes trifoliata, Andromeda polifolia, Sphagnum sp., 9.VII.2008, leg. L.R. \& V.S.

COMMENTS. Teriberka is the northernmost known distribution of the species.

RANGE. West Palaearctic boreo-nemoral.

Agyneta nigripes (Simon, 1884)

MATERIAL. Zonal: $1 \sigma^{7}$, Dal'niye Zelentsy, lichen tundra on top of hill, pitfall traps, 4-26.VII.2009, leg. A.B.

RANGE. Holarctic arcto-alpine.

Allomengea vidua (L. Koch, 1879)

2004 Allomengea vidua - Shokhin et al.: 143, examined.

PREVIOUS RECORDS. Teriberka [Shokhin et al., 2004].

COMMENTS. Teriberka is the northernmost known distribution of the species.

RANGE. Holarctic.

Bathyphantes gracilis (Blackwall, 1841)

MATERIAL. Intra: $1 \sigma^{\top}, 1+$, Teriberka, Sphagnum-Carex bog in tundra, 3.VII.2008, leg. L.R. \& V.S.; $1 \sigma^{7}, 1$ q, CarexSphagnum-Eriophorum bog with Salix sp. stands \& Comarum palustre, 6.VII.2008, leg. L.R. \& V.S.; 1 ๙', Dal'niye Zelentsy, swampy bank of lake, pitfall traps, 4-26.VII.2009, leg. A.B.

RANGE. Holarctic polyzonal.

Bolephthyphantes index (Thorell, 1856)

1912 Bolephthyphantes index - Fedotov: 461

REMARKS. This species is absent from the material examined.

PREVIOUS RECORDS. Murmansk Field Station [Fedotov, 1912].

COMMENTS. This is the northernmost known distribution of the species.

RANGE. Palaearctic boreal-nemoral. Introduced to Greenland [Marusik et. al., 2006].

Bolyphantes luteolus (Blackwall, 1833)

1912 Bolyphantes luteolus - Fedotov: 461.

REMARKS. This species is absent from the material examined.

PREVIOUS RECORDS. Bolshoy Aynovskiy Island, Rybachiy Peninsula, Lodeynaya Bay [Fedotov, 1912].

COMMENTS. Bolshoy Aynovskiy Island is the northernmost known distribution of the species.

RANGE. Palaearctic boreal-nemoral. 1873)

Centromerus arcanus (O. Pickard-Cambridge,

2004 Centromerus arcanus - Shokhin et al.: 143, examined. 
MATERIAL. Zonal: 3 ${ }^{\circ}$, Teriberka, undershrub-lichen mountain tundra with Betula nana, Vaccinium myrtillus, Empetrum sp., Pleurozium schreberi, 4-6.VIII.2009, leg. V.P. Intra: 3 우, elfin Betula sp. sparse forest on hill slope with Vaccinium myrtillus, Gymnocarpium dryopteris, Cornus suecica, Calamagrostis sp., no moss or lichen, 10.VII.2008, leg. L.R. \& V.S.; $1 \overbrace{}^{7}, 3$ 우, Orlovka River valley, elfin Betula sp. sparse forest on gentle slope with Vaccinium myrtillus, Gymnocarpium dryopteris, Cornus suecica no moss or lichen, 9.VII.2008, leg. L.R. \& V.S.; 3 우, Orlovka River valley, swampy meadow with Betula nana, Carex sp., Eriophorum sp., Vaccinium oxycoccus, Rubus chamaemorus, Menyanthes trifoliata, Andromeda polifolia, Sphagnum sp., 9.VII.2008, leg. L.R. \& V.S.; $1 \sigma^{7}$, Dal'niye Zelentsy, Betula sp. sparse forest, pitfall traps, 8-26.VII.2009, leg. A.B. Anthro: 6 우, Teriberka, Betula tortuosa undergrowth on airfield with Empetrum sp., Vaccinium myrtillus, Pleurozium schreberi, Hylocomium sp., etc., 5.VII.2008, leg. L.R. \& V.S.; 5 우, ruderal plant community with Poa sp., Deschampsia caespitosa, Epilobium angustifolium, etc. 3.VII.2008, leg. L.R. \& V.S.

PREVIOUS RECORDS. Teriberka [Shokhin et al., 2004].

COMMENTS. Teriberka is the northernmost known distribution of the species.

RANGE. Palaearctic polyzonal.

Ceratinella brevipes (Westring, 1851)

MATERIAL. Intra: 1 , Dal'niye Zelentsy, Salix sp. with Sphagnum sp. in depression on slope, pitfall traps, 4-26.VII.2009, leg. A.B. Azonal: 1 , meadow-like community on slope to sea, pitfall traps, 4-26.VII.2009, leg. A.B.

COMMENTS. This is the northernmost known distribution of the species.

RANGE. Palaearctic polyzonal.

Ceratinella brevis (Wider, 1834)

MATERIAL. Intra: 1 q, Teriberka, Orlovka River valley, swampy meadows with Betula nana, Carex sp., Eriophorum sp. Vaccinium oxycoccus, Rubus chamaemorus, Menyanthes trifoliata, Andromeda polifolia, Sphagnum sp., 10.VII.2008, leg. L.R. \& V.S.

COMMENTS. Teriberka is the northernmost known distribution of the species. A record of $C$. brevis from Taimyr [Osipov, 2003] is misidentification (material examined).

RANGE. Palaearctic polyzonal.

Ceratinella scabrosa (O. Pickard-Cambridge, 1871)

MATERIAL. Intra: 1 , Teriberka, Orlovka River valley, swampy meadow with Betula nana, Carex sp., Eriophorum sp., Vaccinium oxycoccus, Rubus chamaemorus, Menyanthes trifoliata, Andromeda polifolia, Sphagnum sp., 9.VII.2008, leg. L.R. \& V.S.; 1 + Sphagnum-Carex bog in tundra, 3.VII.2008, leg. L.R. \& V.S.

COMMENTS. Teriberka is the northernmost known distribution of the species.

RANGE. West Palaearctic polyzonal.

Cnephalocotes obscurus (Blackwall, 1834) ined.

2004 Cnephalocotes obscurus - Shokhin et al.: 143, exam-

MATERIAL. Anthro: $2 \sigma^{\top} \sigma^{\top}$, Teriberka, Betula tortuosa undergrowth on airfield with Empetrum sp., Vaccinium myrtillus, Pleurozium schreberi, Hylocomium sp., etc., 4-6.VIII.2009, leg. V.P.
PREVIOUS RECORDS. Teriberka [Shokhin et al, 2004].

COMMENTS. Teriberka is the northernmost known distribution of the species.

RANGE. Holarctic polyzonal.

Diplocephalus cristatus (Blackwall, 1833)

MATERIAL. Anthro: $1 \sigma^{\top}, 1$, Teriberka, meadow in village with Calamagrostis sp., Epilobium angustifolium, Matricaria recutita, Taraxacum sp., 11.VII.2008, leg. L.R. \& V.S.

COMMENTS. This is the northernmost known distribution of the species.

RANGE. Holarctic boreo-nemoral. 1872)

Drepanotylus uncatus (O. Pickard-Cambridge,

2004 Erigoninae - Shokhin et al.: 143, examined.

MATERIAL. Intra: $1 \sigma^{7}$, Teriberka, Sphagnum-Carex bog in tundra, 4-6.VIII.2009, leg. V.P.; $1 \sigma^{7}$, Carex-Sphagnum-Eriophorum bog with Salix sp. stands, Comarum palustre, 6.VII.2008, leg. L.R. \& V.S.; 2 +o, Dal'niye Zelentsy, sedge-sphagnum peatbog, pitfall traps, 4-26.VII.2009, leg. A.B.; 1 \%, Salix sp. with Sphagnum sp. in depression on slope, pitfall traps, 4-26.VII.2009, leg. A.B.

PREVIOUS RECORDS. Teriberka [Shokhin et al., 2004, as "Erigoninae"].

COMMENTS. Teriberka is the northernmost known distribution of the species.

RANGE. West Palaearctic.

Erigone arctica maritima Kulczyński, 1902

1912 Erigone arctica - Fedotov: 457.

2004 E. arctica - Shokhin et al:: 143, examined.

MATERIAL. Intra: 2 우, Dal'niye Zelentsy, sandy bank of lake, pitfall traps, 8-16.VII.2009, leg. A.B. Azonal: 1 , Teriberka, Orlovka River bay, seacoast marsh, under trash \& seaweeds (Fucus sp., Laminaria sp.), 10.VII.2008, leg. L.R. \& V.S. Anthro: 2 우, agricultural meadow with Deschampsia caespitosa, Phleum pratense, Epilobium angustifolium, etc., 4.VII.2008, leg. L.R. \& V.S.; $1 \mathrm{\sigma}$, agricultural meadow with Deschampsia caespitosa, Phleum pratense, Epilobium angustifolium, etc., 4.-6.VIII.2009, leg. V.P.

PREVIOUS RECORDS. Srednyaya Bay, Murmansk Field Station [Fedotov, 1912, as Erigone a. (White, 1852)]; Teriberka [Shokhin et al., 2004, as E. a.].

COMMENTS. Teriberka is the northernmost known distribution of the species.

RANGE. European.

\section{Erigone atra Blackwall, 1833}

1912 Erigone atra - Fedotov: 456.

MATERIAL. Anthro: 2 ${ }_{+}$, Teriberka, agricultural meadow with Deschampsia caespitosa, Phleum pratense, Epilobium angustifolium, etc., 4.VII.2008, leg. L.R. \& V.S.; 1 +, ruderal plant community with Poa sp., Deschampsia caespitosa, Epilobium angustifolium, etc., 3.VII.2008, leg. L.R. \& V.S.; $3 \sigma^{7} \sigma^{7}, 4$ +o, meadow in village with Calamagrostis sp., Epilobium angustifolium, Matricaria recutita, Taraxacum sp., 11.VII.2008, leg. L.R. \& V.S.; 1 , agricultural meadow with Deschampsia caespitosa, Phleum pratense, Epilobium angustifolium, etc., 4-6.VIII.2009, leg. V.P.

PREVIOUS RECORDS. Olen'ya Bay, Srednyaya Bay [Fedotov, 1912].

RANGE. Holarctic polyzonal. 
Erigone tirolensis L. Koch, 1872

1912 Erigone tirolensis - Fedotov: 454.

REMARKS. This species is absent from the material examined.

PREVIOUS RECORDS. Olen'ya Bay [Fedotov, 1912].

RANGE. Holarctic arcto-alpine.

Gonatium rubens (Blackwall, 1833)

MATERIAL. Zonal: $1 q$, Teriberka, near Tretie Titovskoye Lake, dwarf birch-lichen mountain tundra with Betula nana, Cladonia sp., 4-6.VIII.2009, leg. V.P.; 1 ऽ', 3 o, Dal'niye Zelentsy, lichen tundra on top of hill, pitfall traps, 4-26.VII.2009, leg. A.B.; $1 \sigma^{\top}, 5$ 우, lichen tundra on slope of hill, pitfall traps, 4-26.VII.2009, leg. A.B.; 1 q, tundra with Betula nana, Vaccinium myrtillus, Empetrum sp. on slope of hill, pitfall traps, 4-26.VII.2009, leg A.B. Intra: $1 \overbrace{}^{\top}$, Dal'niye Zelentsy, meadow with Geranium sp. in Betula sp. \& Salix sp. stands, pitfall traps, 13-26.VII.2009, leg. A.B. Azonal: $1 \sigma^{7}$, meadow-like community on slope to sea, pitfall traps, 4-26.VII.2009, leg. A.B. Anthro: 1 , Teriberka, Betula tortuosa undergrowth on airfield with Empetrum sp., Vaccinium myrtillus, Pleurozium schreberi, Hylocomium sp., etc., 5.VII.2008, leg. L.R. \& V.S.; 1 + , meadow-like community on wasteland with Epilobium angustifolium, Calamagrostis sp., Polytrichum sp., 7.VII.2008, leg. L.R. \& V.S.; 1 \%, stony wasteland with Deschampsia caespitosa, Phleum pratense, Empetrum sp. \& Cladonia sp. plots, 4-6.VIII.2009, leg. V.P.

RANGE. Palaearctic polyzonal. 1871)

Gongylidiellum latebricola (O. Pickard-Cambridge,

MATERIAL. Intra: $1 \sigma^{\top}, 1$, Teriberka, Orlovka River valley, swampy meadow with Betula nana, Carex sp., Eriophorum sp., Vaccinium oxycoccus, Rubus chamaemorus, Menyanthes trifoliata, Andromeda polifolia, Sphagnum sp., 9.VII.2008, leg. L.R. \& V.S.

COMMENTS. Teriberka is the northernmost known distribution of the species.

RANGE. European polyzonal.

Hilaira herniosa (Thorell, 1875)

2004 Hilaira herniosa - Shokhin et al.: 143, examined.

MATERIAL. Intra: 1 , Dal'niye Zelentsy, meadow with $G e$ ranium sp. in Betula sp. \& Salix sp. stands, pitfall traps, 1326.VII.2009, leg. A.B.

RECORDS. Teriberka [Shokhin et al., 2004].

RANGE. Holarctic boreal.

Hilaira nubigena Hull, 1911

2004 Hilaira sp. - Shokhin et al.: 143, examined.

MATERIAL. Intra: 1 , Teriberka, Sphagnum-Carex bog in tundra, 3.VII.2008, leg. L.R. \& V.S.; 3 우, Dal'niye Zelentsy, sedge-sphagnum peatbog, pitfall traps, 4-26.VII.2009, leg. A.B.

PREVIOUS RECORDS. Teriberka [Shokhin et al., 2004, as Hilaira sp.].

RANGE. Palaearctic-Alaskan boreal.

Hypomma bituberculatum (Wider, 1834)

1912 Dicyphus bituberculatus - Fedotov: 454. ined.

2004 Hypomma bituberculatum - Shokhin et al.: 143, exam-
MATERIAL. Intra: $1 \sigma^{x}, 2$ 우, Teriberka, Orlovka River valley, elfin Betula sp. sparse forest on gentle slope with Vaccinium myrtillus, Gymnocarpium dryopteris, Cornus suecica, no moss or lichen, 9.VII.2008, leg. L.R. \& V.S.; $20^{7} \sigma^{7}, 3$ 9 , Orlovka River valley, elfin Betula sp. sparse forest on gentle slope with Vaccinium myrtillus, Gymnocarpium dryopteris, Cornus suecica, no moss or lichen, 9.VII.2008, leg. L.R. \& V.S.; $1 \sigma^{7}, 8$ 우, SphagnumCarex bog in tundra, 3.VII.2008, leg. L.R. \& V.S.; 2 우, Dal'niye Zelentsy, sedge-sphagnum peatbog, pitfall traps, 4-26.VII.2009, leg. A.B.; $1 O^{7}$, sedge-sphagnum peatbog, pitfall traps, 426.VII.2009, leg. A.B.; $3 \bigcirc^{7} \odot^{7}, 1$, swampy bank of lake, pitfall traps, 4-26.VII.2009, leg. A.B.; $20^{7} \sigma^{7}$, meadow with Geranium sp. in Betula sp. \& Salix sp. stands, pitfall traps, 13-26.VII.2009, leg. A.B. Azonal: $2 \sigma^{\top} \sigma^{\top}, 2$ 우, Dal'niye Zelentsy, meadow-like community on slope to sea, pitfall traps, 4-26.VII.2009, leg. A.B. Anthro: 1 , Teriberka, agricultural meadow with Deschampsia caespitosa, Phleum pratense, Epilobium angustifolium, etc., 4.VII.2008, leg. L.R. \& V.S.; $1 O^{7}, 13$, ruderal plant community with Poa sp., Deschampsia caespitosa, Epilobium angustifolium, etc., 3.VII.2008, leg. L.R. \& V.S.

PREVIOUS RECORDS. Murmansk Field Station [Fedotov, 1912, as Dicyphus b.]; Teriberka [Shokhin et al., 2004].

RANGE. Palaearctic polyzonal.

Hypselistes jacksoni (O. Pickard-Cambridge, 1902)

MATERIAL. Zonal: $1+$, Dal'niye Zelentsy, lichen tundra on top of hill, pitfall traps, 4-26.VII.2009, leg. A.B.; 2 ㅇ, lichen tundra on slope of hill, pitfall traps, 4-26.VII.2009, leg. A.B.; 3 우, tundra with Betula nana, Vaccinium myrtillus, Empetrum sp. on slope of hill, pitfall traps, 4-26.VII.2009, leg. A.B. Intra: $2 \sigma^{7} \sigma^{\top}$, Teriberka, Sphagnum-Carex bog in tundra, 3.VII.2008, leg. L.R. \& V.S.

RANGE. Palaearctic-W-Nearctic boreo-nemoral.

Kaestneria pullata (O. Pickard-Cambridge, 1863)

MATERIAL. Intra: $1+$, Teriberka, Sphagnum-Carex bog in tundra, 3.VII.2008, leg. L.R. \& V.S.

RANGE. Holarctic polyzonal.

Leptorhoptrum robustum (Westring, 1851) ined.

2004 Leptorhoptrum robustum - Shokhin et al.: 143, exam-

MATERIAL. Intra: 1 フ', Dal'niye Zelentsy, meadow with Geranium sp. in Betula sp. \& Salix sp. stands, pitfall traps, 1326.VII.2009, leg. A.B.; 2 +क, Salix sp. with Sphagnum sp. in depression on slope, pitfall traps, 4-26.VII.2009, leg. A.B. Anthro: 1 , Teriberka, agricultural meadow with Deschampsia caespitosa, Phleum pratense, Epilobium angustifolium, etc., 46.VIII.2009, leg. V.P.; 1 9, agricultural meadow on airfield with Deschampsia caespitosa \& Epilobium angustifolium, 5.VII.2008, leg. L.R. \& V.S.

PREVIOUS RECORDS. Teriberka [Shokhin et al., 2004].

COMMENTS. Teriberka is the northernmost known distribution of the species.

RANGE. Palaearctic boreo-nemoral. 1875)

Macrargus multesimus (O. Pickard-Cambridge,

MATERIAL. Zonal: 19 , Teriberka, dwarf birch-lichen mountain tundra with Betula nana, Cladonia sp., 6.VII.2008, leg. L.R. \& V.S.

RANGE. Holarctic boreal. 
Maso sundevalli (Westring, 1851)

MATERIAL. Intra: 1 , Teriberka, Orlovka River valley, elfin Betula sp. sparse forest on gentle slope with Vaccinium myrtillus, Cornus suecica, Geranium sanguineum, Epilobium angustifolium, Calamagrostis sp., 9.VII.2008, leg. L.R. \& V.S.; 2 우, SphagnumCarex bog in tundra, 3.VII.2008, leg. L.R. \& V.S. Anthro: $10^{7}$, Betula tortuosa undergrowth on airfield with Empetrum sp., Vaccinium myrtillus, Pleurozium schreberi, Hylocomium sp., etc. 5.VII.2008, leg. L.R. \& V.S.

COMMENTS. Teriberka is the northernmost known distribution of the species.

RANGE. Holarctic polyzonal.

\section{Mecynargus borealis (Jackson, 1930)}

MATERIAL. Anthro: $1 \sigma^{7}, 1$, Teriberka, agricultural meadow on airfield with Deschampsia caespitosa \& Epilobium angustifolium, 5.VII.2008, leg. L.R. \& V.S.

RANGE. Holarctic boreal.

Mecynargus monticola (Holm, 1943)

MATERIAL. Zonal: 6 우, Teriberka, near Titovskoye Lake, dwarf birch-lichen mountain tundra with Betula nana, Cladonia sp., 4-6.VIII.2009, leg. V.P. Intra: 2 + ${ }^{\text {, Carex-Sphagnum-Erio- }}$ phorum bog with Salix sp. stands, 4-6.VIII.2009, leg. V.P.

RANGE. Palaearctic-W-Nearctic boreal.

Mecynargus morulus (O. Pickard-Cambridge, 1873)

2004 Semljicola faustus - Shokhin et al.: 143, examined.

MATERIAL. Zonal: 1 , Teriberka, undershrub-moss tundra with Empetrum sp., Rubus chamaemorus, Pleurozium schreberi, 4.VII.2008, leg. L.R. \& V.S.; $1 \sigma^{\top}, 1$, undershrub-lichen mountain tundra with Betula nana, Vaccinium myrtillus, Empetrum sp., Pleurozium schreberi, 7.VII.2008, leg. L.R. \& V.S.; 1 O', 1 ㅇ, dwarf birch-lichen mountain tundra with Betula nana, Cladonia sp. 12.VII.2008, leg. L.R. \& V.S.; $2 \sigma^{\top} \sigma^{\top}, 2$ 우, undershrub-moss-lichen mountain tundra with Empetrum sp., Vaccinium myrtillus, Pleurozium schreberi, Dicranum sp., Cladonia sp., 10.VII.2008, leg. L.R. $\&$ V.S.; 1 , undershrub-lichen mountain tundra with Empetrum sp. Arctous alpina, Cladonia sp., no moss or lichen, 9.VII.2008, leg. L.R. \& V.S.; $4 \bigcirc^{\top} \sigma^{\top}, 3$ 90 , near Titovskoye Lake, dwarf birch-lichen mountain tundra with Betula nana, Cladonia sp., 4-6.VIII.2009, leg. V.P.; $1 \sigma^{7}$, undershrub-moss-lichen mountain tundra with Betula nana, Empetrum sp., Pleurozium schreberi, Cladonia sp., Cetraria sp., 4-6.VIII.2009, leg. V.P.; $3 \sigma^{7} \sigma^{7}, 4$ 우, near Tretie Titovskoye Lake, dwarf birch-lichen mountain tundra with Betula nana, Cladonia sp., 4-6.VIII.2009, leg. V.P.; 2 우, Sphagnum-Carex bog in tundra, 3.VII.2008, leg. L.R. \& V.S. Intra: 4 +o, Teriberka, Orlovka River valley, swampy meadow with Betula nana, Carex sp., Eriophorum sp., Vaccinium oxycoccus, Rubus chamaemorus, Menyanthes trifoliata, Andromeda polifolia, Sphagnum sp., 9.VII.2008, leg. L.R. \& V.S. Anthro: $1 \checkmark^{7}, 1$ \%, Teriberka, agricultural meadow on airfield with Deschampsia caespitosa \& Epilobium angustifolium 5.VII.2008, leg. L.R. \& V.S.; $2 \bigcirc^{\top} \bigcirc^{\top}, 2$, 2 , meadow-like community on wasteland with Epilobium angustifolium, Calamagrostis sp., Polytrichum sp., 7.VII.2008, leg. L.R. \& V.S.; 1 + , stony wasteland with Deschampsia caespitosa, Phleum pratense, Empetrum sp. \& Cladonia sp. plots, 4-6.VIII.2009, leg. V.P.

PREVIOUS RECORDS. Teriberka [Shokhin et al., 2004, as Semljicola faustus (O. Pickard-Cambridge, 1900)].

COMMENTS. Teriberka is the northernmost known distribution of the species.

RANGE. European boreo-nemoral.
Mecynargus paetulus (O. Pickard-Cambridge, 1875)

MATERIAL. Anthro: $1 \sigma^{\top}, 1$, Teriberka, agricultural meadow with Deschampsia caespitosa, Phleum pratense, Epilobium angustifolium, etc., 4-6.VIII.2009, leg. V.P.

RANGE. Holarctic boreo-nemoral.

Mecynargus sphagnicola (Holm, 1939)

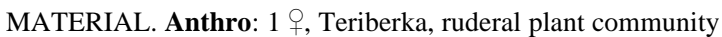
with Poa sp., Deschampsia caespitosa, Epilobium angustifolium, etc., 3.VII.2008, leg. L.R. \& V.S.

RANGE. Fennoscandian-Siberian-W-Nearctic boreal. 1872)

Metopobactrus prominulus (O. Pickard-Cambridge,

MATERIAL. Zonal: $1 \stackrel{q}{q}$, Teriberka, undershrub-moss tundra with Empetrum sp., Rubus chamaemorus, Pleurozium schreberi, 4-6.VIII.2009, leg. V.P.; 1 ऽ', Dal'niye Zelentsy, lichen tundra on top of hill, pitfall traps, 4-26.VII.2009, leg. A.B.

RANGE. Holarctic polyzonal.

Micrargus herbigradus (Blackwall, 1854)

MATERIAL. Anthro: 2 q+, Teriberka, ruderal plant community with Poa sp., Deschampsia caespitosa, Epilobium angustifolium, etc., 3.VII.2008, leg. L.R. \& V.S.

COMMENTS. Teriberka is the northernmost known distribution of the species.

RANGE. Palaearctic boreo-nemoral.

Minicia marginella (Wider, 1834)

MATERIAL. Anthro: 19 , Teriberka, Betula tortuosa undergrowth on airfield with Empetrum sp., Vaccinium myrtillus, Pleurozium schreberi, Hylocomium sp., etc., 4-6.VIII.2009, leg. V.P.

COMMENTS. Teriberka is the northernmost known distribution of the species.

RANGE. Palaearctic polyzonal. 1863)

Minyrioloides trifrons (O. Pickard-Cambridge,

MATERIAL. Intra: $1 \Im^{r}, 2$, 9 , Teriberka, Orlovka River valley, elfin Betula sp. sparse forest on gentle slope with Vaccinium myrtillus, Cornus suecica, Geranium sanguineum, Epilobium angustifolium, Calamagrostis sp., 9.VII.2008, leg. L.R. \& V.S.

COMMENTS. Teriberka is the northernmost known distribution of the species.

RANGE. Holarctic boreo-nemoral.

Minyriolus pusillus (Wider, 1834)

MATERIAL. Anthro: $1 \uparrow$, Teriberka, ruderal plant community with Poa sp., Deschampsia caespitosa, Epilobium angustifolium, etc., 3.VII.2008, leg. L.R. \& V.S.; 1 q, Betula tortuosa undergrowth on airfield with Empetrum sp., Vaccinium myrtillus, Pleurozium schreberi, Hylocomium sp., etc., 4-6.VIII.2009, leg. V.P.

COMMENTS. Teriberka is the northernmost known distribution of the species.

RANGE. Palaearctic boreo-nemoral. 
Oedothorax gibbosus (Blackwall, 1841)

MATERIAL. Intra: 1 ', Teriberka, Carex-Sphagnum-Eriophorum bog with Salix sp. stands, Comarum palustre, 6.VII.2008 leg. L.R. \& V.S.

COMMENTS. Teriberka is the northernmost known distribution of the species.

RANGE. West Palaearctic polyzonal.

Oedothorax retusus (Westring, 1851)

1912 Kulczynskiellum retusum - Fedotov: 454.

2004 Oedothorax apicatus - Shokhin et al.: 143, examined.

MATERIAL. Zonal: $1 \sigma^{\gamma}$, Teriberka, undershrub-moss tundra with Empetrum sp., Rubus chamaemorus, Pleurozium schreberi, 4.VII.2008, leg. L.R. \& V.S.; 2 +o, Dal'niye Zelentsy, lichen tundra on slope of hill, pitfall traps, 4-26.VII.2009, leg. A.B. Intra: $1 \sigma^{7}$, Teriberka, Orlovka River valley, swampy meadows with Betula nana, Carex sp., Eriophorum sp., Vaccinium oxycoccus, Rubus chamaemorus, Menyanthes trifoliata, Andromeda polifolia, Sphag num sp. 10.VII.2008, leg. L.R. \& V.S.; $2 \sigma^{7} \sigma^{7}, 1$ o, Dal'niye Zelentsy, flood peddle bank of river, pitfall traps, 8-20.VII.2009, leg. A.B.; $1 \overbrace{}^{7}$, swampy bank of lake, pitfall traps, 4-26.VII.2009, leg. A.B.; 4 옹 Salix sp. with Sphagnum sp. in depression on slope, pitfall traps, 4-26.VII.2009, leg. A.B. Azonal: 1 , Dal'niye Zelentsy, meadow-like plant community on slope to sea, pitfall traps, 4-14.VII.2009, leg. A.B. Anthro: $1 \sigma^{7}, 4$ 00 , Teriberka, rudera plant community with Poa sp., Deschampsia caespitosa, Epilobium angustifolium, etc., 3.VII.2008, leg. L.R. \& V.S.; $1 \sigma^{7}, 1$, agricultural meadow with Deschampsia caespitosa, Phleum pratense, Epilobium angustifolium, etc., 4.VII.2008, leg. L.R. \& V.S.; 2 우, agricultural meadow on airfield with Deschampsia caespitosa \& Epilobium angustifolium, 5.VII.2008, leg. L.R. \& V.S.; 1 , meadow in village with Calamagrostis sp., Epilobium angustifolium, Matricaria recutita, Taraxacum sp., 11.VII.2008, leg. L.R. \& V.S.

PREVIOUS RECORDS. Pala Bay [Fedotov, 1912, as Kulczynskiellum r.]; Teriberka [Shokhin et al., 2004, as Oedothorax apicatus (Blackwall, 1850)].

RANGE. Palaearctic polyzonal.

\section{Oreoneta sinuosa (Tullgren, 1955)}

1912 Hilaira frigida - Fedotov: 457.

2004 Hilaira tatrica - Shokhin et al.: 143, examined.

MATERIAL. Anthro: 1 , Teriberka, stony wasteland with Deschampsia caespitosa, Phleum pratense, Empetrum sp. \& Cladonia sp. plots, 5.VII.2008, leg. L.R. \& V.S.; 1 , agricultural meadow on airfield with Deschampsia caespitosa \& Epilobium

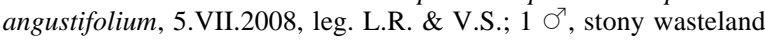
with Deschampsia caespitosa, Phleum pratense, Empetrum sp. \& Cladonia sp. plots, 4-6.VIII.2009, leg. V.P.

PREVIOUS RECORDS. Srednyaya Bay [Fedotov, 1912, as Hilaira frigida (Thorell, 1872)]. Teriberka [Shokhin et al., 2004, as H. tatrica Kulczyński, 1915].

COMMENTS. Teriberka is the northernmost known distribution of the species.

RANGE. Fennoscandian.

Oreonetides vaginatus (Thorell, 1872)

1912 Oreonetides vaginatus - Fedotov: 458.

2004 Saaristoa abnormis - Shokhin et al.: 143, examined.

PREVIOUS RECORDS. Olen'ya Bay, Pala Bay

[Fedotov, 1912]; Teriberka [Shokhin et al., 2004, as Saaristoa abnormis (Blackwall, 1841)].

RANGE. Holarctic boreal.
Oryphantes angulatus (O. Pickard-Cambridge, 1881)

1912 Lepthyphantes sp. - Fedotov: 459, pl. 8: 8, 9.

1913 L. murmanicola Strand: 42, syn.n.

1942 L. obscurus Fedotov. - Roewer: 551, incorrect citation.

2004 L. angulatus - Shokhin et al.: 143, examined.

MATERIAL. Intra: 3 of, Teriberka, elfin Betula sp. sparse forest on hill slope with Vaccinium myrtillus, Gymnocarpium dryopteris, Cornus suecica, Calamagrostis sp., no moss or lichen, 10.VII.2008, leg. L.R. \& V.S.; 1 +, Orlovka River valley, swampy meadow with Betula nana, Carex sp., Eriophorum sp., Vaccinium oxycoccus, Rubus chamaemorus, Menyanthes trifoliata, Andromeda polifolia, Sphagnum sp., 9.VII.2008, leg. L.R. \& V.S.; 2 +o, Sphagnum-Carex bog in tundra, 3.VII.2008, leg. L.R. \& V.S.; $1 \sigma^{7}$, Dal'niye Zelentsy, swampy bank of lake, pitfall traps, 426.VII.2009, leg. A.B.

PREVIOUS RECORDS. Rybachiy Peninsula, Zemlyanoye [Fedotov, 1912, as Lepthyphantes sp.]; Teriberka [Shokhin et al., 2004, as L. a.].

TAXONOMIC REMARKS. In his paper on the spiders of Murmansk Area, Fedotov [1912] reported on and illustrated a single female of Lepthyphantes sp. from Rybachiy Peninsula and noted that the species would probably be a new species similar to $L$. angulipalpis (Westring, 1851). Later on, this species was named by Strand [1913] as L. murmanicola Strand, 1913. According to the original figures by Fedotov [1912: pl. 8: 8, 9], the latter author unambiguously dealt with a female of Oryphantes angulatus, and therefore it is safe to conclude that the name L. murmanico$l a$ is to be treated as its junior synonym (syn.n.). The findings of $O$. angulatus in the regions close to the $L$. murmanicola type locality [see Shokhin et al., 2004 and present paper] further support this conclusion.

COMMENTS. Rybachiy Peninsula is the northernmost known distribution of the species.

RANGE. European boreo-nemoral.

Pelecopsis mengei Simon, 1926

2004 Pelecopsis elongata - Shokhin et al.: 143, examined.

MATERIAL. Intra: $2 \sigma^{7} \sigma^{7}, 8$ 우, Teriberka, Orlovka River valley, elfin Betula sp. sparse forest on gentle slope with Vaccinium myrtillus, Cornus suecica, Geranium sanguineum, Epilobium angustifolium, Calamagrostis sp., 9.VII.2008, leg. L.R. \& V.S.; 1 q, Orlovka River valley, elfin Betula sp. sparse forest on gentle slope with Vaccinium myrtillus, Gymnocarpium dryopteris, Cornus suecica, no moss or lichen, 9.VII.2008, leg. L.R. \& V.S.; 1 , Sphagnum-Carex bog in tundra, 3.VII.2008, leg. L.R. \& V.S.; 1 , Orlovka River valley, swampy meadows with Betula nana, Carex sp., Eriophorum sp., Vaccinium oxycoccus, Rubus chamaemorus, Menyanthes trifoliata, Andromeda polifolia, Sphagnum sp. 10.VII.2008, leg. L.R. \& V.S.; 1 +, Dal'niye Zelentsy, sedge-sphagnum peatbog, pitfall traps, 4-26.VII.2009, leg. A.B.; 2 우, meadow with Geranium sp. in Betula sp. \& Salix sp. stands, pitfall traps, 1326.VII.2009, leg. A.B.; $1 \sigma^{7}$, Betula sp. sparse forest, pitfall traps, 8 26.VII.2009, leg. A.B. Azonal: $3 \circ$, Dal'niye Zelentsy, meadow-like community on slope to sea, pitfall traps, 4-26.VII.2009, leg. A.B. Anthro: 1 , Teriberka, Betula tortuosa undergrowth on airfield with Empetrum sp., Vaccinium myrtillus, Pleurozium schreberi, Hylocomium sp., etc., 5.VII.2008, leg. L.R. \& V.S.; $1 \sigma^{7}, 1$ ㅇ, ruderal plant community with Poa sp., Deschampsia caespitosa, Epilobium angustifolium, etc., 3.VII.2008, leg. L.R. \& V.S.

PREVIOUS RECORDS. Teriberka [Shokhin et al., 2004, as Pelecopsis elongata (Wider, 1834)].

RANGE. Holarctic polyzonal. 


\section{Pocadicnemis pumila (Blackwall, 1841)}

2004 Pocadicnemis pumila - Shokhin et al.: 143, examined. MATERIAL. Intra: $5 \sigma^{\top} \sigma^{\top}, 26$ 우, Teriberka, elfin Betula sp. sparse forest on hill slope with Vaccinium myrtillus, Gymnocarpium dryopteris, Cornus suecica, Calamagrostis sp., no moss or lichen, 9-10.VII.2008, leg. L.R. \& V.S.

PREVIOUS RECORDS. Teriberka [Shokhin et al., 2004].

COMMENTS. Teriberka is the northernmost known distribution of the species.

RANGE. Holarctic polyzonal.

Poeciloneta variegata (Blackwall, 1841)

1912 Poeciloneta variegata - Fedotov: 458.

REMARKS. This species is absent from the material examined.

PREVIOUS RECORDS. Murmansk Field Station [Fedotov, 1912].

COMMENTS. This is the northernmost known distribution of the species.

RANGE. Palaearctic-W-Nearctic polyzonal.

Porrhomma convexum (Westring, 1851)

1912 Porrhomma norvegicum - Fedotov: 457.

REMARKS. This species is absent from the material examined.

PREVIOUS RECORDS. Pala Bay [Fedotov, 1912, as Porrhomma norvegicum Strand, 1901].

COMMENTS. This is the northernmost known distribution of the species.

RANGE. European polyzonal. All records of $P$. convexum in the Nearctic require confirmation.

\section{Porrhomma egeria Simon, 1884}

2004 Porrhomma convexum - Shokhin et al.: 143, examined. PREVIOUS RECORDS. Teriberka [Shokhin et al., 2004, as Porrhomma convexum].

COMMENTS. This is the northernmost known distribution of the species.

RANGE. European boreo-nemoral.

Semljicola barbiger (L. Koch, 1879)

MATERIAL. Zonal: 19 , Teriberka, undershrub-moss tundra with Empetrum sp., Rubus chamaemorus, Pleurozium schreberi, 4-6.VIII.2009, leg. V.P. Intra: $1 \sigma^{7}$, Dal'niye Zelentsy, swampy bank of lake, pitfall traps, 4-26.VII.2009, leg. A.B.

RANGE. Fennoscandian-Siberian arcto-boreal.

Semljicola faustus (O. Pickard-Cambridge, 1900)

MATERIAL. Azonal: $1 \sigma^{r}$, Dal'niye Zelentsy, meadow-like community on slope to sea, pitfall traps, 4-26.VII.2009, leg. A.B.

COMMENTS. This is the northernmost known distribution of the species.

RANGE. Fennoscandian-Siberian boreal.

Semljicola lapponicus (Holm, 1939)

MATERIAL. Intra: 2 우, Teriberka, Sphagnum-Carex bog in tundra, 4-6.VIII.2009, leg. V.P.; $2 \sigma^{\top} \sigma^{\top}, 2$ 우, Carex-Sphagnum-
Eriophorum bog with Salix sp. stands, 4-6.VIII.2009, leg. V.P. Anthro: $1+$, Teriberka, agricultural meadow with Deschampsia caespitosa, Phleum pratense, Epilobium angustifolium, etc., 4.6.VIII.2009, leg. V.P.

RANGE. Fennoscandian-Siberian-W-Nearctic boreal.

Silometopus reussi (Thorell, 1871)

MATERIAL. Anthro: $1 \sigma^{7}, 7$ 우, Teriberka, agricultural meadow with Deschampsia caespitosa, Phleum pratense, Epilobium angustifolium, etc., 4.VII.2008, leg. L.R. \& V.S.

COMMENTS. This is the northernmost known distribution of the species.

RANGE. Palaearctic boreo-nemoral.

Sisicus apertus (Holm, 1939)

MATERIAL. Zonal: 19 , Teriberka, undershrub-lichen mountain tundra with Betula nana, Vaccinium myrtillus, Empetrum sp., Pleurozium schreberi, 4-6.VIII.2009, leg. V.P.

COMMENTS. This is the northernmost known distribution of the species.

RANGE. Holarctic boreo-nemoral.

Tapinocyba pallens (O. Pickard-Cambridge, 1872)

MATERIAL. Intra: 3 o+, Teriberka, elfin Betula sp. sparse forest on hill slope with Vaccinium myrtillus, Gymnocarpium dryopteris, Cornus suecica, Calamagrostis sp., no moss or lichen, 10.VII.2008, leg. L.R. \& V.S.

COMMENTS. This is the northernmost known distribution of the species.

RANGE. European boreo-nemoral.

Tenuiphantes alacris (Blackwall, 1853)

1912 Lepthyphantes alacris - Fedotov: 459.

2004 L. alacris - Shokhin et al.: 143, examined.

MATERIAL. Azonal: $1 \sigma^{7}, 1$, Dal'niye Zelentsy, meadow-like community on slope to sea, pitfall traps, 4-26.VII.2009, leg. A.B.

PREVIOUS RECORDS. Murmansk Field Station [Fedotov, 1912, as Lepthyphantes a.]; Teriberka [Shokhin et al., 2004, as L. a.].

COMMENTS. Teriberka is the northernmost known distribution of the species.

RANGE. Palaearctic polyzonal.

Tenuiphantes nigriventris (L. Koch, 1879)

MATERIAL. Intra: $1 \sigma^{r}$, Teriberka, Orlovka River valley, elfin Betula sp. sparse forest on gentle slope with Vaccinium myrtillus, Cornus suecica, Geranium sanguineum, Epilobium angustifolium, Calamagrostis sp., 9.VII.2008, leg. L.R. \& V.S.; 1 , Orlovka River valley, elfin Betula sp. sparse forest on gentle slope with Vaccinium myrtillus, Gymnocarpium dryopteris, Cornus sueci$c a$, no moss or lichen, 9.VII.2008, leg. L.R. \& V.S.

COMMENTS. Teriberka is the northernmost known distribution of the species.

RANGE. Holarctic boreo-nemoral.

Tenuiphantes tenebricola (Wider, 1834)

MATERIAL. Anthro: $1 \sigma^{r}, 1$, Teriberka, ruderal plant community with Poa sp., Deschampsia caespitosa, Epilobium angustifolium, etc., 3.VII.2008, leg. L.R. \& V.S. 
COMMENTS. This is the northernmost known distribution of the species.

RANGE. Palaearctic boreo-nemoral.

Tiso aestivus (L. Koch, 1872)

MATERIAL. Zonal: $1 \sigma^{7}$, Dal'niye Zelentsy, lichen tundra on top of hill, pitfall traps, 4-26.VII.2009, leg. A.B.; Anthro: 1 Teriberka, meadow-like community on wasteland with Epilobium angustifolium, Calamagrostis sp., Polytrichum sp., 7.VII.2008, leg. L.R. \& V.S.

RANGE. Holarctic polyzonal.

Wabasso replicatus (Holm, 1950)

MATERIAL. Intra: $1 \sigma^{\top}$, Teriberka, Carex-Sphagnum-Eriophorum bog with Salix sp. stands, Comarum palustre, 6.VII.2008, leg. L.R. \& V.S.; 2 +क, Carex-Sphagnum-Eriophorum bog with Salix sp. stands, 4-6.VIII.2009, leg. V.P.

RANGE. West Palaearctic boreal.

Walckenaeria capito (Westring, 1861)

2004 Walckenaeria sp. - Shokhin et al.: 143, examined.

MATERIAL. Anthro: 1 o, Teriberka, Betula tortuosa undergrowth on airfield with Empetrum sp., Vaccinium myrtillus, Pleurozium schreberi, Hylocomium sp., etc., 4-6.VIII.2009, leg. V.P.

PREVIOUS RECORDS. Teriberka [Shokhin et al., 2004, as Walckenaeria sp.].

COMMENTS. This is the northernmost known distribution of the species.

RANGE. Holarctic polyzonal.

Walckenaeria cuspidata Blackwall, 1833

2004 Walckenaeria sp. - Shokhin et al.: 143, examined.

MATERIAL. Intra: 3 우, Dal'niye Zelentsy, meadow with Geranium sp. in Betula sp. \& Salix sp. stands, pitfall traps, 13 26.VII.2009, leg. A.B. Azonal: 1 , meadow-like community on slope to sea, pitfall traps, 4-26.VII.2009, leg. A.B.

PREVIOUS RECORDS. Teriberka [Shokhin et al., 2004, as Walckenaeria. sp.].

COMMENTS. Teriberka is the northernmost known distribution of the species.

RANGE. Holarctic polyzonal. 1873)

Walckenaeria karpinskii (O. Pickard-Cambridge, ined.

2004 Walckenaeria karpinskii - Shokhin et al.: 143, exam-

MATERIAL. Zonal: 1 ऽ, Teriberka, near Titovskoye Lake, dwarf birch-lichen mountain tundra with Betula nana, Cladonia sp., 4-6.VIII.2009, leg. V.P.; $3 \sigma^{7} \sigma^{7}, 4$ 우, near Tretie Titovskoye Lake, dwarf birch-lichen mountain tundra with Betula nana, Cladonia sp., 4-6.VIII.2009, leg. V.P. 1 \%, Dal'niye Zelentsy, lichen tundra on slope of hill, pitfall traps, 4-26.VII.2009, leg. A.B.; 1 , tundra with Betula nana, Vaccinium myrtillus, Empetrum sp. on slope of hill, pitfall traps, 4-26.VII.2009, leg. A.B. Anthro: 1 , Teriberka, ruderal plant community with Poa sp., Deschampsia caespitosa, Epilobium angustifolium, etc., 3.VII.2008, leg. L.R. \& V.S.

PREVIOUS RECORDS. Teriberka [Shokhin et al., 2004].

RANGE. Fennoscandian-Siberian-Nearctic boreal.
Walckenaeria kochi (O. Pickard-Cambridge, 1872)

MATERIAL. Intra: $1+$, Teriberka, Carex-Sphagnum-Eriophorum bog with Salix sp. stands, 4-6.VIII.2009, leg. V.P.

COMMENTS. This is the northernmost known distribution of the species.

RANGE. Palaearctic boreo-nemoral.

Walckenaeria mitrata (Menge, 1868)

2004 Walckenaeria mitrata - Shokhin et al.: 143, examined. PREVIOUS RECORDS. Teriberka [Shokhin et al., 2004].

COMMENTS. This is the northernmost known distribution of the species.

RANGE. European boreo-nemoral.

Walckenaeria nudipalpis (Westring, 1851) ined.

2004 Walckenaeria nudipalpis — Shokhin et al.: 143, exam-

MATERIAL. Anthro: 2 +⿱ , Teriberka, ruderal plant community with Poa sp., Deschampsia caespitosa, Epilobium angustifolium, etc., 3.VII.2008, leg. L.R. \& V.S

PREVIOUS RECORDS. Teriberka [Shokhin et al., 2004].

COMMENTS. This is the northernmost known distribution of the species.

RANGE. Palaearctic polyzonal.

Zornella cultrigera (L. Koch, 1879)

2004 Erigoninae - Shokhin et al.: 143, examined.

MATERIAL. Intra: $1 \sigma^{7}, 1$, Teriberka, Orlovka River valley, elfin Betula sp. sparse forest on gentle slope with Vaccinium myrtillus, Gymnocarpium dryopteris, Cornus suecica, no moss or lichen, 9.VII.2008, leg. L.R. \& V.S.; 2 90, Dal'niye Zelentsy, Betula sp. sparse forest, pitfall traps, 8-26.VII.2009, leg. A.B. Anthro: 1 , Teriberka, ruderal plant community with Poa sp., Deschampsia caespitosa, Epilobium angustifolium, etc., 3.VII.2008, leg. L.R. \& V.S.

PREVIOUS RECORDS. Teriberka [Shokhin et al., 2004, as "Erigoninae"].

RANGE. Holarctic boreal.

Fam. TETRAGNATHIDAE (1)

Tetragnatha extensa (Linnaeus, 1758)

1912 Tetragnatha extensa - Fedotov: 461.

REMARKS. This species is absent from the material examined.

PREVIOUS RECORDS. Olen'ya Bay [Fedotov, 1912].

RANGE. Holarctic polyzonal.

Fam. ARANEIDAE (3)

Araneus diadematus Clerck, 1757

1912 Araneus diadematus - Fedotov: 462.

REMARKS. This species is absent from the material examined. 
PREVIOUS RECORDS. Murmansk Field Station [Fedotov, 1912].

RANGE. Holarctic polyzonal.

Larinioides cornutus (Clerck, 1757)

1912 Araneus cornutus — Fedotov: 462.

REMARKS. This species is absent from the material examined.

PREVIOUS RECORDS. Olen'ya Bay [Fedotov, 1912, as Araneus c.].

RANGE. Holarctic polyzonal.

Larinioides patagiatus (Clerck, 1757)

1912 Araneus patagiatus - Fedotov: 462.

REMARKS. This species is absent from the material examined.

PREVIOUS RECORDS. Murmansk Field Station,

Pala Bay [Fedotov, 1912, as Araneus p.].

RANGE. Holarctic polyzonal.

Fam. LYCOSIDAE (15)

Alopecosa aculeata (Clerck, 1757)

1912 Tarentula aculeata - Fedotov: 464

2004 T. aculeata - Shokhin et al: 142

MATERIAL. Intra: $3 \sigma^{\top} \sigma^{\top}$, Dal'niye Zelentsy, Salix sp. with Sphagnum sp. in depression on slope, pitfall traps, 4-26.VII.2009, leg. A.B

PREVIOUS RECORDS. Murmansk Field Station, Tyuva Bay [Fedotov, 1912, as Tarentula a.]; Teriberka [Shokhin et al., 2004, as T. a.].

RANGE. Holarctic polyzonal.

Arctosa alpigena (Doleschall, 1852)

1912 Arctosa alpigena - Fedotov: 466

2004 Tricca alpigena - Shokhin et al.: 142.

MATERIAL. Zonal: 1 q, Teriberka, undershrub-moss-lichen mountain tundra with Betula nana, Empetrum sp., Pleurozium schreberi, Cladonia sp., Cetraria sp. 4.VII.2008, leg. L.R. \& V.S.; 1 ㅇ, near Tretie Titovskoye Lake, dwarf birch-lichen mountain tundra with Betula nana, Cladonia sp., 4-6.VIII.2009, leg. V.P.; 1 , Dal'niye Zelentsy, lichen tundra on slope of hill, pitfall traps, 4 11.VII.2009, leg. A.B. Intra: $5 \sigma^{\top} \sigma^{\top}, 3$ sedge-sphagnum peatbog, pitfall traps, 4-26.VII.2009, leg. A.B.; ${ }^{\top}, 1$, meadow with Geranium sp. in Betula sp. \& Salix sp. stands, pitfall traps, 13-26.VII.2009, leg. A.B.; $2 \sigma^{7} \sigma^{7}, 1$, sedgesphagnum peatbog, pitfall traps, 4-26.VII.2009, leg. A.B. Azonal: $2 \sigma^{7} \sigma^{7}, 3$ 우, Dal'niye Zelentsy, meadow-like community on slope to sea, pitfall traps, 4-26.VII.2009, leg. A.B. Anthro: 1 , Teriberka, ruderal plant community with Poa sp., Deschampsia caespitosa, Epilobium angustifolium, etc., 3.VII.2008, leg. L.R. \& V.S

PREVIOUS RECORDS. Lodeynaya Bay [Fedotov, 1912]; Teriberka [Shokhin et al., 2004, as Tricca a.].

RANGE. Holarctic polyzonal.

Pardosa agrestis (Westring, 1861)

2004 Pardosa monticola - Shokhin et al.: 142, not seen.

REMARKS. This species is absent from the material examined.
PREVIOUS RECORDS. Teriberka [Shokhin et al., 2004].

COMMENTS. A record of $P$. monticola (Clerck, 1757) most probably refers to $P$. agrestis. For details, see Zyuzin [1979].

RANGE. Palaearctic polyzonal.

Pardosa agricola (Thorell, 1856)

MATERIAL. Zonal: 1 , Teriberka, undershrub-moss tundra with Empetrum sp., Rubus chamaemorus, Pleurozium schreberi, 4.VII.2008, leg. L.R. \& V.S.

COMMENTS. This is the northernmost known distribution of the species.

RANGE. European boreo-nemoral.

Pardosa amentata (Clerck, 1757)

1912 Lycosa saccata - Fedotov: 467.

2004 Pardosa amentata - Shokhin et al.: 142.

MATERIAL. Intra: $8 \bigcirc^{\top} \sigma^{\top}, 7$ 우, Dal'niye Zelentsy, Salix sp. with Sphagnum sp. in depression on slope, pitfall traps, 426.VII.2009, leg. A.B.; $1 \sigma^{7}$, swampy bank of lake, pitfall traps, 4 26.VII.2009, leg. A.B.; 2 우, Betula sp. sparse forest, pitfall traps, 8-26.VII.2009, leg. A.B.; $12 \sigma^{\top} \sigma^{\top}, 2$ O+ , sandy bank of lake, pitfall traps, 8-16.VII.2009, leg. A.B. Anthro: 1 \%, Teriberka, agricultural meadow with Deschampsia caespitosa, Phleum pratense, Epilobium angustifolium, etc., 4.VII.2008, leg. L.R. \& V.S.; 1 \%, ruderal plant community with Poa sp., Deschampsia caespitosa, Epilobium angustifolium, etc., 3.VII.2008, leg. L.R. \& V.S.

PREVIOUS RECORDS. Shalim Island, Pala Bay, Srednyaya Bay, Tyuva Bay [Fedotov, 1912, as Lycosa saccata (Linnaeus, 1758)], Teriberka [Shokhin et al., 2004].

COMMENTS. Shalim Island is the northernmost known distribution of the species.

RANGE. European boreo-nemoral.

Pardosa atrata (Thorell, 1873)

2004 Pardosa atrata - Shokhin et al.: 142

MATERIAL. Intra: $15 \sigma^{\top} \sigma^{7}, 14$ 우, Dal'niye Zelentsy, sedgesphagnum peatbog, pitfall traps, 4-26.VII.2009, leg. A.B.; 2 우, swampy bank of lake, pitfall traps, 4-26.VII.2009, leg. A.B.; 4 $\Im^{\top} \circlearrowleft^{\top}, 4$ 우, sedge-sphagnum peatbog, pitfall traps, 4-26.VII.2009, leg. A.B.

PREVIOUS RECORDS. Teriberka [Shokhin et al., 2004].

COMMENTS. Teriberka is the northernmost known distribution of the species.

RANGE. Palaearctic boreo-nemoral.

Pardosa eiseni (Thorell, 1875)

2004 Pardosa eiseni - Shokhin et al.: 142.

MATERIAL. Intra: $1 \circ$, Dal'niye Zelentsy, meadow with $G e$ ranium sp. in Betula sp. \& Salix sp. stands, pitfall traps, 1326.VII.2009, leg. A.B. Azonal: 1 , meadow-like community on slope to sea, pitfall traps, 4-14.VII.2009, leg. A.B. 2004]

PREVIOUS RECORDS. Teriberka [Shokhin et al.,

COMMENTS. Teriberka is the northernmost known distribution of the species.

RANGE. Palaearctic boreal. 
Pardosa hyperborea (Thorell, 1872)

1912 Lycosa hyperborea - Fedotov: 470.

2004 Pardosa hyperborea - Shokhin et al.: 142.

MATERIAL. Intra: $1 \sigma^{7}$, Teriberka, Orlovka River valley, swampy meadow with Betula nana, Carex sp., Eriophorum sp., Vaccinium oxycoccus, Rubus chamaemorus, Menyanthes trifoliata, Andromeda polifolia, Sphagnum sp., 9.VII.2008, leg. L.R. \& V.S.; 1 $\sigma^{7}$, Orlovka River valley, swampy meadows with Betula nana, Carex sp., Eriophorum sp., Vaccinium oxycoccus, Rubus chamaemorus, Menyanthes trifoliata, Andromeda polifolia, Sphagnum sp. 10.VII.2008, leg. L.R. \& V.S.; $5 \sigma^{\top} \sigma^{\top}$, Dal'niye Zelentsy, Salix sp. with Sphagnum sp. in depression on slope, pitfall traps, 4 11.VII.2009, leg. A.B.; $1 \sigma^{7}$, flood pebble bank of river, pitfall traps, 8-20.VII.2009, leg. A.B. Anthro: 2 우, Betula tortuosa undergrowth on airfield with Empetrum sp., Vaccinium myrtillus, Pleurozium schreberi, Hylocomium sp., etc., 5.VII.2008, leg. L.R. \& V.S.

PREVIOUS RECORDS. Murmansk Field Station, Olen'ya Bay, Lodeynaya Bay [Fedotov, 1912, as Lycosa h.]; Teriberka [Shokhin et al., 2004].

RANGE. Holarctic boreo-nemoral.

Pardosa lasciva L. Koch, 1879

MATERIAL. Intra: $1 \bigcirc^{\top}, 1$, Dal'niye Zelentsy, flood peddle bank of river, pitfall traps, 8-20.VII.2009, leg. A.B.

COMMENTS. Dal'niye Zelentsy is the northernmost known distribution of the species.

RANGE. Palaearctic boreal.

Pardosa lugubris (Walckenaer, 1802)

1912 Lycosa chelata - Fedotov: 468

MATERIAL. Intra: 1 , Teriberka, Orlovka River valley, elfin Betula sp. sparse forest on gentle slope with Vaccinium myrtillus, Gymnocarpium dryopteris, Cornus suecica, no moss or lichen, 9.VII.2008, leg. L.R. \& V.S.; 1 フ', Orlovka River valley, swampy meadow with Betula nana, Carex sp., Eriophorum sp., Vaccinium oxycoccus, Rubus chamaemorus, Menyanthes trifoliata, Andromeda polifolia, Sphagnum sp., 9.VII.2008, leg. L.R. \& V.S. Anthro: $1 \sigma^{7}$, meadow in village with Calamagrostis sp., Epilobium angustifolium, Matricaria recutita, Taraxacum sp., 11.VII.2008, leg. L.R. \& V.S.

PREVIOUS RECORDS. Shalim Island, Tyuva Bay [Fedotov, 1912, as Lycosa chelata (Müller, 1764)].

COMMENTS. Shalim Island is the northernmost known distribution of the species.

RANGE. Palaearctic polyzonal.

Pardosa palustris (Linnaeus, 1758)

1912 Pardosa tarsalis - Fedotov: 468.

2004 Lycosa palustris - Shokhin et al.: 143, examined.

MATERIAL. Zonal: $1 \sigma^{7}$, Teriberka, undershrub-moss tundra with Empetrum sp., Rubus chamaemorus, Pleurozium schreberi, 4.VII.2008, leg. L.R. \& V.S.; ca $200 \sigma^{\top} \sigma^{7}, 15$ 우, Dal'niye Zelentsy, tundra with Betula nana, Vaccinium myrtillus, Empetrum sp. on slope of hill, pitfall traps, 4-26.VII.2009, leg. A.B.; $56 \sigma^{\top} \sigma^{\top}$, 11 우, lichen tundra on slope of hill, pitfall traps, 4-26.VII.2009, leg. A.B.; $14 \bigcirc^{\top} \sigma^{7}, 3$ 우, lichen tundra on top of hill, pitfall traps, 4-26.VII.2009, leg. A.B. Intra: $5 \sigma^{\top} \sigma^{\top}$, Dal'niye Zelentsy, sedgesphagnum peatbog, pitfall traps, 4-26.VII.2009, leg. A.B.; $20 \bigcirc^{7} \sigma^{7}$, Betula sp. sparse forest, pitfall traps, 8-26.VII.2009, leg. A.B.; 18 $\sigma^{\top} \sigma^{\top}, 1$, flood pebble bank of river, pitfall traps, 8-20.VII.2009, leg. A.B.; $5 \sigma^{7} \sigma^{7}$, swampy bank of lake, pitfall traps, 4-26.VII.2009, leg. A.B.; $21 \sigma^{7} \sigma^{7}, 3$ 0 , meadow with Geranium sp. in Betula sp. \& Salix sp. stands, pitfall traps, 13-26.VII.2009, leg. A.B.; 50 $\sigma^{7} \sigma^{\top}, 1$, Salix sp. with Sphagnum sp. in depression on slope, pitfall traps, 4-26.VII.2009, leg. A.B.; $22 \sigma^{\top} \sigma^{\gamma}, 4$ 우, sandy bank of lake, pitfall traps, 8-16.VII.2009, leg. A.B.; 23 o $\sigma^{7}$, sedgesphagnum peatbog, pitfall traps, 4-26.VII.2009, leg. A.B. Azonal: $220 \Im^{7} \sigma^{\top}, 25$ of , meadow-like community on slope to sea, pitfall traps, 4-26.VII.2009, leg. A.B. Anthro: 1 을 Teriberka, agricultural meadow on airfield with Deschampsia caespitosa \& Epilobium angustifolium, 5.VII.2008, leg. L.R. \& V.S.

PREVIOUS RECORDS. Bolshoy Aynovskiy Island, Rybachiy Peninsula, Yekaterininskiy Island, Murmansk Field Station, Lodeynaya Bay, Karabelnaya Bay [Fedotov, 1912, as Lycosa tarsalis (Thorell, 1856)]; Teriberka [Shokhin et al., 2004].

RANGE. Holarctic polyzonal.

Pardosa prativaga (L. Koch, 1870)

REMARKS. This species is absent from the material examined. 1912].

PREVIOUS RECORDS. Olen'ya Bay [Fedotov,

RANGE. Palaearctic polyzonal.

Pardosa sphagnicola (F. Dahl, 1908)

1912 Lycosa riparia subsp. sphagnicola - Fedotov: 467.

REMARKS. This species is absent from the material examined.

PREVIOUS RECORDS. Olen'ya Bay [Fedotov, 1912, as Lycosa riparia subsp. sphagnicola Dahl, 1908]. RANGE. Palaearctic polyzonal.

Pirata piraticus (Clerck, 1757)

MATERIAL. Intra: $1 \overbrace{}^{7}$, Teriberka, Carex-Sphagnum-Eriophorum bog with Salix sp. stands, Comarum palustre, 6.VII.2008, leg. L.R. \& V.S.; $1 \sigma^{7}$, Dal'niye Zelentsy, swampy bank of lake, pitfall traps, 4-26.VII.2009, leg. A.B.

RANGE. Holarctic polyzonal.

Pirata uliginosus (Thorell, 1856)

MATERIAL. Intra: $1 \sigma^{\top}$, Teriberka, Orlovka River valley, swampy meadow with Betula nana, Carex sp., Eriophorum sp., Vaccinium oxycoccus, Rubus chamaemorus, Menyanthes trifoliata, Andromeda polifolia, Sphagnum sp., 9.VII.2008, leg. L.R. \& V.S.

RANGE. Holarctic polyzonal.

Fam. AGELENIDAE (1)

Tegenaria domestica (Clerck, 1757)

1912 Tegenaria derhami - Fedotov: 465.

REMARKS. This species is absent from the material examined.

PREVIOUS RECORDS. Sedlovaty Island [Fedotov, 1912, as Tegenaria derhami Scopoli, 1763].

RANGE. Cosmopolitan domestic.

Fam. HAHNIIDAE (2)

Cryphoeca silvicola (C.L. Koch, 1834)

1912 Cryphoeca silvicola - Fedotov: 465.

MATERIAL. Zonal: $1 \uparrow$, Teriberka, near Tretie Titovskoye Lake, dwarf birch-lichen mountain tundra with Betula nana, Cladonia sp., 4-6.VIII.2009, leg. V.P. 
PREVIOUS RECORDS. Kildin Island, Lodeynaya Bay [Fedotov, 1912].

RANGE. Palaearctic boreo-nemoral.

Hahnia ononidum Simon, 1875

2004 Hahnia ononidum - Shokhin et al.: 143.

MATERIAL. Zonal: 1 , Dal'niye Zelentsy, lichen tundra on slope of hill, pitfall traps, 4-26.VII.2009, leg. A.B.; $2 \sigma^{\top} \sigma^{\top}, 3$ 90 , tundra with Betula nana, Vaccinium myrtillus, Empetrum sp. on slope of hill, pitfall traps, 4-26.VII.2009, leg. A.B.; 1 ऽ', 1 \%, Betula sp. sparse forest, pitfall traps, 8-26.VII.2009, leg. A.B. Intra: $1 \sigma^{7}$, Teriberka, Orlovka River valley, elfin Betula sp. sparse forest on gentle slope with Vaccinium myrtillus, Cornus suecica, Geranium sanguineum, Epilobium angustifolium, Calamagrostis sp., 9.VII.2008, leg. L.R. \& V.S.; 1 フ , Dal'niye Zelentsy, Salix sp. with Sphagnum sp. in depression on slope, pitfall traps, 4-11.VII.2009, leg. A.B. Azonal: $1 \sigma^{7}$, Dal'niye Zelentsy, meadow-like plant community on slope to sea, pitfall traps, 4-14.VII.2009, leg. A.B.

PREVIOUS RECORDS. Teriberka [Shokhin et al., 2004].

RANGE. Holarctic boreo-nemoral.

Fam. DICTINIDAE (1)

Dictyna arundinacea (Linnaeus, 1758)

1912 Dictyna arundinacea - Fedotov: 451.

REMARKS. This species is absent from the material examined.

PREVIOUS RECORDS. Murmansk Field Station [Fedotov, 1912].

RANGE. Holarctic polyzonal.

Fam. CLUBIONIDAE (3)

Clubiona germanica Thorell, 1871

1912 Clubiona germanica - Fedotov: 465.

REMARKS. This species is absent from the material examined.

PREVIOUS RECORDS. Srednyaya Bay [Fedotov, 1912].

RANGE. Palaearctic polyzonal.

Clubiona kulczynskii Lessert, 1905

1912 Clubiona borealis — Fedotov: 464.

REMARKS. This species is absent from the material examined.

PREVIOUS RECORDS. Srednyaya Bay, Tyuva Bay [Fedotov, 1912, as Clubiona borealis Thorell, 1871].

RANGE. Holarctic polyzonal.

Clubiona norvegica Strand, 1900

MATERIAL. Intra: $1 \sigma^{7}$, Teriberka, Sphagnum-Carex bog in tundra, 3.VII.2008, leg. L.R. \& V.S

RANGE. Holarctic.

Fam. GNAPHOSIDAE (4)

Gnaphosa microps Holm, 1939

MATERIAL. Zonal: 1 , Teriberka, undershrub-moss tundra with Empetrum sp., Rubus chamaemorus, Pleurozium schreberi, 4.VII.2008, leg. L.R. \& V.S
RANGE. Holarctic boreal.

Micaria alpina L. Koch, 1872

2004 Micaria alpina - Shokhin et al.: 143.

REMARKS. This species is absent from the material examined.

PREVIOUS RECORDS. Teriberka [Shokhin et al., 2004].

RANGE. Holarctic polyzonal.

Micaria pulicaria (Sundevall, 1831)

2004 Micaria pulicaria - Shokhin et al.: 143.

REMARKS. This species is absent from the material examined.

PREVIOUS RECORDS. Teriberka [Shokhin et al., 2004].

RANGE. Holarctic polyzonal.

Haplodrassus soerenseni (Strand, 1900)

1912 Drassodes soerenseni - Fedotov: 452.

REMARKS. This species is absent from the material examined.

PREVIOUS RECORDS. Lodeynaya Bay [Fedotov, 1912 as Drassodes s.].

RANGE. Palaearctic boreal.

Fam. PHILODROMIDAE (1)

Thanatus arcticus Thorell, 1872

1912 Thanatus arcticus - Fedotov: 463.

REMARKS. This species is absent from the material examined.

PREVIOUS RECORDS. Murmansk Field Station [Fedotov, 1912].

RANGE. Holarctic arcto-boreal.

Fam. THOMISIDAE (4)

Ozyptila arctica Kulczyński, 1908

2004 Ozyptila sp. - Shokhin et al.: 143, examined.

MATERIAL. Intra: 1 , Teriberka, Orlovka River valley, swampy meadows with Betula nana, Carex sp., Eriophorum sp., Vaccinium oxycoccus, Rubus chamaemorus, Menyanthes trifoliata, Andromeda polifolia, Sphagnum sp. 10.VII.2008, leg. L.R. \& V.S.; 1 ㅇ, Sphagnum-Carex bog in tundra, 4-6.VIII.2009, leg. V.P. Azonal: $1+$, Dal'niye Zelentsy, meadow-like community on slope to sea, pitfall traps, 4-26.VII.2009, leg. A.B.

PREVIOUS RECORDS. Teriberka [Shokhin et al., 2004, as Ozyptila sp.].

RANGE. Fennoscandian-Siberian-Nearctic arctoboreal.

Ozyptila trux (Blackwall, 1846)

MATERIAL. Intra: $1 \sigma^{7}, 4$ $9 \circ$, Teriberka, Orlovka River valley, elfin Betula sp. sparse forest on gentle slope with Vaccinium myrtillus, Cornus suecica, Geranium sanguineum, Epilobium angustifolium, Calamagrostis sp., 9.VII.2008, leg. L.R. \& V.S.; 1 o', Orlovka River valley, swampy meadow with Betula nana, 
Carex sp., Eriophorum sp., Vaccinium oxycoccus, Rubus chamaemorus, Menyanthes trifoliata, Andromeda polifolia, Sphagnum sp., 9.VII.2008, leg. L.R. \& V.S.; 1 9, Sphagnum-Carex bog in tundra, 3.VII.2008, leg. L.R. \& V.S. Anthro: $1 \sigma^{7}, 2$ 우, rudera plant community with Poa sp., Deschampsia caespitosa, Epilobium angustifolium, etc., 3.VII.2008, leg. L.R. \& V.S.

RANGE. Palaearctic polyzonal.

Xysticus albidus Grese, 1909

MATERIAL. Zonal: $4 \sigma^{7} \sigma^{7}, 1$, Dal'niye Zelentsy, lichen tundra on top of hill, pitfall traps, 4-26.VII.2009, leg. A.B.

RANGE. Fennoscandian-Siberian boreal.

Xysticus cristatus (Clerck, 1757)

2004 Xysticus cristatus - Shokhin et al.: 143, examined.

MATERIAL. Anthro: 1 \%, Teriberka, Betula tortuosa undergrowth on airfield with Empetrum sp., Vaccinium myrtillus, Pleurozium schreberi, Hylocomium sp., etc., 4-6.VIII.2009, leg. V.P. 2004].

PREVIOUS RECORDS. Teriberka [Shokhin et al.,

RANGE. Palaearctic polyzonal.

Fam. SALTICIDAE (3)

Evarcha arcuata (Clerck, 1757)

1912 Evarcha arcuata - Fedotov: 472.

REMARKS. This species is absent from the material examined.

PREVIOUS RECORDS. Yekaterininskiy Island [Fedotov, 1912].

RANGE. Palaearctic polyzonal.

Evarcha falcata (Clerck, 1757)

1912 Evarcha falcata - Fedotov: 472.

REMARKS. This species is absent from the material examined.

PREVIOUS RECORDS. Pala Bay [Fedotov, 1912].

RANGE. Palaearctic polyzonal.

Sitticus pubescens (Fabricius, 1775)

1912 Sitticus pubescens - Fedotov: 472.

REMARKS. This species is absent from the material examined.

PREVIOUS RECORDS. Lodeynaya Bay [Fedotov, 1912].

RANGE. Holarctic polyzonal.

\section{Discussion}

Thus, 105 species of spiders have been found in a tundra zone of the Kola Peninsula. The total is less than half that found in a tundra belt of the Russian Plain [see Tanasevitch \& Koponen, 2007]. The reason is possibly attributable to the fact that the area of the Kola tundra is smaller than tundra area in the European plain and is not under the strong influence of the Siberian fauna. Further, the tundra zone of the Eastern Europe is penetrated by rivers flowing in a meridional direction, which supplies the southern elements in the tundra zone. Numerous species are moving northward in the wide valleys; the tundra parts do not fall outside the limits of valleys or river terraces, thus some southern elements enter on watersheds and occupy not only the intrazonal communities, but also even become elements of the zonal fauna.

The Kola tundra is not a continuous, uniform belt. It is appreciably fragmented by the strong vertical partitioning of the landscape, and the presence of numerous lakes and rivers which break and mask the zonality. To allocate a zonal faunistic kernel in the fauna of the Kola tundra, we have divided the investigated biotopes as zonal, intrazonal and azonal. These three biotopes correspond to certain types of vegetation (see above). We have also allocated anthropogenically modified biotopes, as being rather specific and very characteristic for the present tundra. Distributions of the spiders by the zonal-landscape types of the plant communities are as presented in Table 1. Unfortunately, not all spider findings of previous authors were accompanied by detailed descriptions of biotopes, thus it is difficult to define now what types of communities spiders have been collected in. Therefore, the table is based mostly on our original data. The table shows that the fauna of the zonal plant communities of the Kola tundra is the poorest. As is known, the zonal tundra communities are the biotopes with most pessimal values of abiotic factors. Therefore, those organisms living in the zonal communities must have specific adaptations for residing there, or have a wide range of ecological tolerances. It is obvious that there are few spider species have either specific adaptations or a wide range of tolerances. Poverty of tundra zonal communities is caused by a lack of vital space for soildwelling spiders and their potential victims: the inhabited layer (moss-lichen cover) is very thin. From the 21 species found in the tundra zonal communities, only one, Agyneta nigripes, is arcto-alpine. The others are typically boreal, e.g., Cryphoeca silvicola, Gnaphosa microps, Macrargus multesimus, Mecynargus monticola, M. morulus, W. karpinskii, etc., or polyzonal and ubiquitous, e.g., Centromerus arcanus, Gonatium rubens, Metopobactrus prominulus, Oedothorax retusus, and Tiso aestivus.

Unlike the extreme or pessimal conditions in the zonal communities, those abiotic factors in the intrazonal communities are smoothed and lacking lower extremes; the moss and leaf litter layer is much thicker than it is in the moss-lichen tundra, therefore, the taxonomic variety and the population density of spiders is much higher here. Thus, the fauna of the intrazonal communities of the tundra zone ( 52 species) is richer than that in the zonal community. Further, the typical boreal and the polyzonal species which have the majority of species, contain southern elements, e.g., Agyneta mossica, Ceratinella scabrosa, Gongylidiellum latebricola, Oryphantes angulatus, and Tapinocyba pallens.

Azonal plant communities occupy an insignificant space in the Kola tundra and have essentially less varia- 
Table 1. Distribution of spiders between zonal-landscape types of plant communities in the Kola tundra. Таблица 1. Зонально-ландшафтное распределение пауков Кольских тундр.

\begin{tabular}{|c|c|c|c|c|}
\hline \multirow[b]{2}{*}{$\begin{array}{c}\text { Таха } \\
\text { Таксоны }\end{array}$} & \multicolumn{4}{|c|}{$\begin{array}{l}\text { Zonal-landscape types of plant communities } \\
\text { Зонально-ландшафтные типы сообществ }\end{array}$} \\
\hline & $\begin{array}{c}\text { Zonal } \\
\text { Зональные }\end{array}$ & $\begin{array}{c}\text { Intrazonal } \\
\text { Интра- } \\
\text { зональные }\end{array}$ & $\begin{array}{c}\text { Azonal } \\
\text { Азональные }\end{array}$ & $\begin{array}{c}\text { Anthropogenically } \\
\text { modified biotopes } \\
\text { Антропогенные } \\
\text { биотопы }\end{array}$ \\
\hline 1 & 2 & 3 & 4 & 5 \\
\hline \multicolumn{5}{|l|}{ Fam. THERIDIIDAE } \\
\hline Robertus lividus (B1., 1836) & & + & + & + \\
\hline R. scoticus Jack., 1914 & + & + & & + \\
\hline \multicolumn{5}{|l|}{ Fam. LINYPHIIDAE } \\
\hline Agyneta conigera (O. P.-Cambr., 1863) & & + & & \\
\hline A. mossica (Schikora, 1993) & & + & & \\
\hline A. nigripes (Simon, 1884) & + & & & \\
\hline Allomengea vidua (L. Koch, 1879) & & + & & \\
\hline Bathyphantes gracilis (Bl., 1841) & & + & & \\
\hline Bolephthyphantes index (Thor., 1856) & & + & & \\
\hline Bolyphantes luteolus (B1.,1833) & & & + & + \\
\hline Centromerus arcanus (O. P.-Cambr., 1873) & + & + & & + \\
\hline Ceratinella brevipes (Westr., 1851) & & + & + & \\
\hline C. brevis (Wid., 1834) & & + & & \\
\hline C. scabrosa (O. P.-Cambr., 1871) & & + & & \\
\hline Cnephalocotes obscurus (Bl., 1834) & & & & + \\
\hline Diplocephalus cristatus (B1., 1833) & & & & + \\
\hline Drepanotylus uncatus (O. P.-Cambr.,1872) & & + & & \\
\hline Erigone arctica maritima Kulcz., 1902 & & + & + & + \\
\hline E. atra $\mathrm{Bl} ., 1833$ & & & + & + \\
\hline E. tirolensis L. Koch, 1872 & & & + & \\
\hline Gonatium rubens (B1., 1833) & + & + & + & + \\
\hline Gongylidiellum latebricola (O. P.-Cambr., 1871) & & + & & \\
\hline Hilaira herniosa (Thor., 1875) & & + & & \\
\hline H. nubigena Hull, 1911 & & + & & \\
\hline Hypomma bituberculatum (Wid., 1834) & & + & + & + \\
\hline Hypselistes jacksoni (O. P.-Cambr., 1902) & + & + & & \\
\hline Kaestneria pullata (O. P.-Cambr., 1863) & & + & & \\
\hline Leptorhoptrum robustum (Westr., 1851) & & + & & + \\
\hline Macrargus multesimus (O. P.-Cambr., 1875) & + & & & \\
\hline Maso sundevalli (Westr., 1851) & & + & & + \\
\hline Mecynargus borealis (Jack., 1930) & & & & + \\
\hline M. monticola (Holm, 1943) & + & + & & \\
\hline M. morulus (O. P.-Cambr., 1873) & + & + & & + \\
\hline M. paetulus (O. P.-Cambr., 1875) & & & & + \\
\hline M. sphagnicola $($ Holm, 1939) & & & & + \\
\hline Metopobactrus prominulus (O. P.-Cambr., 1872) & + & & & \\
\hline Micrargus herbigradus (B1., 1854) & & & & + \\
\hline Minicia marginella (Wid., 1834) & & & & + \\
\hline Minyrioloides trifrons (O. P.-Cambr., 1863) & & + & & \\
\hline Minyriolus pusillus (Wid., 1834) & & & & + \\
\hline Oedothorax gibbosus (B1., 1841) & & & + & \\
\hline O. retusus (Westr., 1851) & + & + & + & + \\
\hline Oreoneta sinuosa (Tullgren, 1955) & & & & + \\
\hline Oreonetides vaginatus (Thor., 1872) & & & + & + \\
\hline Oryphantes angulatus (O. P.-Cambr., 1881) & & + & + & \\
\hline Pelecopsis mengei Simon, 1926 & & + & + & + \\
\hline Pocadicnemis pumila (B1., 1841) & & + & & \\
\hline Poeciloneta variegata (B1., 1841) & & & + & \\
\hline
\end{tabular}


Table 1 (continuing) Таблица 1 (продолжение)

\begin{tabular}{|c|c|c|c|c|}
\hline 1 & 2 & 3 & 4 & 5 \\
\hline Porrhomma convexum (Westr., 1851) & & & & + \\
\hline \multicolumn{5}{|l|}{ P. egeria Simon, 1884} \\
\hline Semljicola barbiger (L. Koch, 1879) & + & + & & \\
\hline S. faustus (O. P.-Cambr., 1900) & & & + & \\
\hline S. lapponicus (Holm, 1939) & & + & & + \\
\hline Silometopus reussi (Thor., 1871) & & & & + \\
\hline Sisicus apertus $($ Holm, 1939) & + & & & \\
\hline Tapinocyba pallens (O. P.-Cambr., 1872) & & + & & \\
\hline Tenuiphantes alacris $(\mathrm{B} 1 ., 1853)$ & & & + & + \\
\hline T. nigriventris (L. Koch, 1879) & & + & & \\
\hline T. tenebricola (Wid., 1834) & & & & + \\
\hline Tiso aestivus (L. Koch, 1872) & + & & & + \\
\hline Wabasso replicatus (Holm, 1950) & & + & & \\
\hline Walckenaeria capito (Westr., 1861) & & & & + \\
\hline W. cuspidata $\mathrm{Bl} ., 1833$ & & + & + & \\
\hline W. karpinskii (O. P.-Cambr., 1873) & + & & & + \\
\hline W. kochi (O. P.-Cambr., 1872) & & + & & \\
\hline \multicolumn{5}{|l|}{ W. mitrata (Menge, 1868) } \\
\hline W. nudipalpis (Westr., 1851) & & & & + \\
\hline Zornella cultrigera (L. Koch, 1879) & & + & & + \\
\hline \multicolumn{5}{|l|}{ Fam. TETRAGNATHIDAE } \\
\hline Tetragnatha extensa $(\mathrm{L} ., 1758)$ & & & + & \\
\hline \multicolumn{5}{|l|}{ Fam. ARANEIDAE } \\
\hline \multicolumn{5}{|l|}{ Araneus diadematus $\mathrm{Cl} ., 1757$} \\
\hline \multicolumn{5}{|l|}{ Larinioides patagiatus (Cl., 1757) } \\
\hline \multicolumn{5}{|l|}{ L. cornutus $(\mathrm{Cl} ., 1757)$} \\
\hline \multicolumn{5}{|l|}{ Fam. LYCOSIDAE } \\
\hline Alopecosa aculeata (Cl., 1757) & & + & & \\
\hline Arctosa alpigena (Dol., 1852) & + & + & + & + \\
\hline \multicolumn{5}{|l|}{ Pardosa agrestis (Westring, 1861) } \\
\hline P. agricola (Thor., 1856) & + & & & \\
\hline P. amentata $(\mathrm{Cl} ., 1757)$ & & + & & + \\
\hline P. atrata (Thor., 1873) & & + & & \\
\hline P. eiseni (Thor., 1875) & & + & + & \\
\hline P. hyperborea (Thor., 1872) & & + & + & + \\
\hline P. lasciva L. Koch, 1879 & & + & & \\
\hline P. lugubris (Walck., 1802) & & + & & + \\
\hline P. palustris (L., 1758) & + & + & + & + \\
\hline \multicolumn{5}{|l|}{ P. prativaga $($ L. Koch, 1870$)$} \\
\hline \multicolumn{5}{|l|}{ P. sphagnicola (F. Dahl, 1908) } \\
\hline Pirata piraticus $(\mathrm{Cl} ., 1757)$ & & + & & \\
\hline P. uliginosus (Thor., 1856) & & + & & \\
\hline \multicolumn{5}{|l|}{ Fam. AGELENIDAE } \\
\hline \multicolumn{5}{|l|}{ Tegenaria domestica $(\mathrm{Cl} ., 1757)$} \\
\hline \multicolumn{5}{|l|}{ Fam. HAHNIIDAE } \\
\hline Cryphoeca silvicola (C.L. Koch, 1834) & + & & & \\
\hline Hahnia ononidum Simon, 1875 & + & + & + & \\
\hline \multicolumn{5}{|l|}{$\begin{array}{ll}\text { Fam. DICTYNIDAE } \\
\end{array}$} \\
\hline \multicolumn{5}{|l|}{ Dictyna arundinacea (L., 1758) } \\
\hline \multicolumn{5}{|l|}{ Fam. CLUBIONIDAE } \\
\hline \multicolumn{5}{|l|}{ Clubiona germanica Thor., 1871} \\
\hline \multicolumn{5}{|l|}{ C. kulczynskii Lessert, 1905} \\
\hline C. norvegica Strand, 1900 & & + & & \\
\hline Fam. GNAPHOSIDAE & & & & \\
\hline Gnaphosa microps Holm, 1939 & + & & & \\
\hline
\end{tabular}


Table 1 (continuing) Таблица 1 (продолжение)

\begin{tabular}{|l|c|c|c|c|}
\hline \multicolumn{1}{|c|}{1} & 2 & 3 & 4 & 5 \\
\hline Haplodrassus soerenseni (Strand, 1900) & & & & \\
\hline Micaria alpina L. Koch, 1872 & & & & \\
\hline M. pulicaria (Sund., 1831) & & & & \\
\hline Fam. PHILODROMIDAE & & & & \\
\hline Thanatus arcticus Thor., 1872 Fam. THOMISIDAE & & & & \\
\hline Ozyptila arctica Kulcz., 1908 & & & & + \\
\hline O. trux (B1., 1846) & & + & & + \\
\hline Xysticus albidus Grese, 1909 & & + & & \\
\hline X. cristatus (Cl., 1757) & + & & & \\
\hline Fam. SALTICIDAE & & & & \\
\hline Evarcha arcuata (Cl., 1757) & & & & \\
\hline E. falcata (Cl., 1757) & & & & \\
\hline Sitticus pubescens (Fabr., 1775) & & & & \\
\hline TOTAL species: & $\mathbf{2 1}$ & $\mathbf{5 2}$ & $\mathbf{2 4}$ & $\mathbf{4 0}$ \\
\hline
\end{tabular}

tion than does the intrazonal. Accordingly, their fauna is poorer ( 24 species). As a specific element, it is possible to note only the arcto-alpine species, Erigone tirolensis.

Anthropogenically modified plant communities and biotopes are very rich with spiders (40 species), but the species here are generalists. That is, they come from many sources (e.g., tundra, Arctic, boreal, polyzonal, Holarctic, southern, etc.). These communities and biotopes are wellwarmed places, good refuges that are protected from winds and precipitation; there is enough space for their webs and there is an abundance of prey. A complete spectrum of the zonal-landscape groups of spiders is represented here, and this is a place for the most probable detection of the introduced species. Some species were found only here, i.e., Cnephalocotes obscurus, Diplocephalus cristatus, Mecynargus sphagnicola, Micrargus herbigradus, Minyriolus pusillus, Porrhomma convexum, Tenuiphantes tenebricola, and Walckenaeria nudipalpis.

As is known in the southern tundra, the intrazonal and azonal communities serve as a shelter not only for southern faunistic elements, but also for Arctic ones. From three arcto-alpine species, only Agyneta nigripes is found in zonal tundra; Ozyptila arctica lives in intrazonal and azonal communities, Erigone tirolensis is found only in azonal communities. In general, the penetration of the Arctic species into southern tundra goes by settling of the intrazonal, azonal and anthropogenically modified biotopes. This has been shown repeatedly by Eskov [1986], Tanasevitch \& Koponen [2007], and the reason for this is not yet clear. It is very strange, but no real Arctic elements have yet been found in Kola Peninsula tundra belt, species such as Halorates holmgreni (Thorell, 1871), H. spetsbergensis (Thorell, 1872), or any other Arctic species that habit the European sector of the Arctic.

\section{Conclusions}

The spider fauna of a tundra landscape zone of the Kola Peninsula has a typical boreal face, and is comprised of boreal and polyzonal European or widespread species. No real Arctic elements are found here. The zonal plant communities have the fewest spider species, as they lack the specific elements and are comprised of boreal, polyzonal or ubiquitous species. The spider fauna of the intrazonal and anthropogenic communities is much richer, and together with azonal communities, they serve as a shelter not only for the southern elements, but also for the Arctic species.

ACKNOWLEDGEMENTS. We are very grateful to Victor B. Semenov, Victor S. Piryugin and Anatoly B. Babenko (Moscow, Russia) whose collections have been used in the present study, as well as to Alexander V. Ponomarev (Rostov-na-Donu, Russia) for comments on Shokhin's collection from Kola Peninsula. Special thanks go to Robin Leech who kindly checked the English of an advanced draft. The study was supported in part by the Russian Foundation for Basic Research, Projects \# 09-04-01365-a and \# 08-04-92230-a.

\section{References}

Chernov Yu.I. 1978. [The structure of the animal population of the Subarctic]. Moscow: Nauka Publ. 165 pp. [in Russian]

Fedotov D.M. 1912. [To the spider fauna of Murman and Novaya Zemlya] // Annu. Mus. Zool. Ac. Sc. St.-Petersb. Vol.16 (1911). No.4. P.449-474 [in Russian].

Marusik Y.M., Bocher J, Koponen S. 2006. The collection of Greenland spiders (Aranei) kept in the Zoological Museum, University of Copenhagen // Arthropoda Selecta. Vol.15. No.1. P.59-80.

Osipov D.V. 2003. [Structure of the spider (Aranei) fauna in southern tundra of western Taimyr] // Zool. zhurn. Vol.82. No.10. P.1266-1270 [in Russian, with English summary].

Shokhin I.V., Ponomaryov A.V., Kiyashko P.V. 2004. [Materials to the fauna of terrestrial invertebrates of Murmansk Area] // Evolyutsiya morskikh i nazemnykh ekosistem v periglyatsialnykh zonakh. Rostov-na-Donu. P.140-143 [in Russian].

Strand E. 1913. Arachnida fur 1912 // Arch. Naturg. Bd.79. H.10. S. 15-61.

Tanasevitch A.V., Koponen S. 2007. Spiders (Aranei) of the southern tundra in the Russian Plain // Arthropoda Selecta. Vol.15. No.4. P.295-345 (for 2006)

Zyuzin A.A. 1979. [A taxonomic study of Palearctic spiders of the genus Pardosa C.L. Koch (Aranei, Lycosidae). I. The taxonomic structure of the genus] // Entomol. obozr. Vol.58. No.2. P.431-447 [in Russian, with English summary]. 